\title{
Structural vibration analysis with random fields using the hierarchical finite element method
}

\author{
A. T. Fabro, ${ }^{a, *}$ N. S. Ferguson ${ }^{b}$, B. R. Mace $^{c}$, \\ ${ }^{a}$ Department of Mechanical Engineering, University of Brasilia, Brasilia, Brazil \\ ${ }^{\mathrm{b}}$ ISVR, University of Southampton, Southampton, UK \\ c Acoustics Research Centre, Department of Mechanical Engineering, University of \\ Auckland, Auckland, New Zealand
}

Keywords: Hierarchical finite element, random field, Karhunen-Loève, structural vibration.

\begin{abstract}
Element based techniques, like the Finite Element method, are the standard approach in industry for low frequency applications in structural dynamics. However, mesh requirements can significantly increase the computational cost for increasing frequencies. In addition, randomness in system properties starts to play a significant role and its inclusion in the model further increases the computational cost. In this paper, a hierarchical finite element formulation is presented which incorporates spatially random properties. Polynomial and trigonometric hierarchical functions are used in the element formulation. Material and geometrical spatially correlated randomness are represented by the Karhunen-Loève expansion, a series representation for random fields. It allows the element integration to be performed only once for each term of the series which has benefits for a sampling scheme and can be used for non-Gaussian distributions. Free vibration and forced response statistics are calculated using the proposed approach. Compared to the standard $h$-version, the hierarchical finite element approach produces smaller mass and stiffness matrices, without changing the number of nodes of the element, and tends to be computationally more efficient. These are key factors not only when considering solutions for higher frequencies but also in the calculation of response statistics using a sampling method such as Monte Carlo simulation.
\end{abstract}

\section{Introduction}

Uncertainty and variability play an important role in structural dynamics such that the standard deterministic Finite Element (FE) method [1,2] by itself is not able to predict the structural dynamics, it being necessary to add some level of random description. Furthermore, a fine mesh is required for mid and high frequency applications [3] in which the pollution effect must also be taken into account, due to a degeneracy of the FE solution for decreasing wavelengths [4], further increasing the computational cost.

Structural uncertainty modelling often involves modelling spatial random variability. It becomes even more relevant when dealing with, for instance, composite materials [5-9]

*Corresponding author

Address: Universidade de Brasília - Campus Darcy Ribeiro, Faculdade de Tecnologia,

Departmento de Engenharia Mecânica, Brasília, DF, 70910-900, +55 6131075682

Email address: fabro@unb.br 
or additive manufacturing [10,11]. One of the available tools to describe spatially correlated variability is the random field [12,13], which is a multidimensional random process using a probability measure. Ideally, the representation should succeed in capturing the essential features of a complex random phenomenon in terms of a small number of physically meaningful and experimentally accessible parameters [12]. Several approaches have been proposed to determine the Representative Volume Element with spatial randomness using homogenization techniques, e.g. [14-17].

Furthermore, the inclusion of uncertainty in the parameters of the mechanical model of a structure has attracted much research interest in recent decades. The stochastic finite element method (SFEM) [18-22] is an extension of deterministic FE in the sense that it incorporates the random parameters in the standard FE formulation. It requires modelling the random fields for material or geometrical properties and its discretization, i.e. an approximation of a continuous random field by a finite number of random variables. State-of-the-art reviews of the SFEM and uncertainty in linear dynamic systems, covering the representation of a random field, its simulation, modelling and implementation in mechanical models, as well as the implementation of the different stochastic solvers can be found in the literature [19,21-23].

Ghanem and Spanos [13] proposed a spectral approach to the SFEM, the spectral stochastic finite element method (SSFEM), in which the response is represented by the coefficients of basis functions defined over the domain of the random variables, a polynomial chaos basis. However, for small correlation lengths, the matrices can became huge [19]. Ostoja-Starzewski and Woods [24] proposed a formulation of the SFEM for vibrating rods and beams using a spectral expansion of random fields. Adhikari [25] extended the weak-form of the FE approach, using analytical solutions for homogeneous rods and beams as shape functions, and the analytical expression of the Karhunen-Loève (KL) expansion of a Gaussian homogeneous random field with a specific correlation function, to produce closed form expressions for the mass, damping and stiffness matrices. However, the formulation is restricted to cases where such an analytical solution is available. Yang et al. [26] proposed a hierarchical formulation for the stochastic FE for static analysis, by using the Krylov subspace method, forming a hierarchical vector basis for the response.

In this work, it is proposed to use the hierarchical finite element (HFE) method [1], also known as the $p$-element formulation, with random fields, in order to reduce computational cost, but keeping the versatility of the FE formulation. The HFE method can also be considered as a special case of the Rayleigh-Ritz method, with a different choice of admissible functions, improving versatility and convergence rate [27]. It consists of enriching the displacement field with a series of polynomials of increasing order, without changing the number of nodes of the element. Different hierarchical shape functions can be used, including trigonometric functions [28], but usually the Rodrigues's form of the Legendre polynomial is applied [29]. As opposed to the FE approach, where mesh refinement is required for solutions at high frequencies and conversely smaller wavelengths, the HFE formulation keeps the mesh size fixed and increases the degree of the approximating functions used in the element formulation, which makes it very 
appealing to energy distribution or power flow calculations [30,31]. It has been used in many applications, including the vibration analysis of beams [32-34], shafts [35], plates and cylinders, including composite laminates or stiffened structures [28,29,36-38]. Compared to the standard $h$-version FE, the HFE method produces smaller mass and stiffness matrices and tends to be computationally more efficient. These are key factors not only when considering the solution at higher frequencies, but also in the calculation of response statistics using a sampling method like Monte Carlo (MC) sampling [39]. In this paper, a stochastic hierarchical finite element (SHFE) formulation is presented for one-dimensional rod and beam elements and two-dimensional flexural thin plate elements. Polynomial and trigonometric hierarchical functions are used in the element formulation. Material and geometrical spatially correlated randomness are included by using the KL expansion, a series representation for random fields in terms of deterministic functions weighted by random coefficients. It can represent both Gaussian and nonGaussian random fields. Therefore, the element integration needs to be performed only once for each term of the series. This approach becomes more important when using a sampling scheme because, unlike the case for which element properties are constant, the element integration step is costly when compared to the solution of the linear system itself. Even though a specific analytical solution of the KL expansion is used in this paper, the proposed formulation can also be applied with numerical solutions for different correlation functions and probability distributions. Free vibration and forced response statistics are calculated using the proposed approach. Although the work is focused on vibration analysis, the proposed approach could also be used for static analysis.

In section 2, an analytical solution of the KL expansion is reviewed for a random field with exponentially decaying correlation function. This solution is used throughout the paper. In section 3, the HFE formulation using the KL expansion is presented and particular results for rod, beam and thin plate elements are derived. In section 4, numerical results are presented, considering the Young's modulus of elasticity as a Gamma distributed random field, for different cases for the correlation length and standard deviation. Finally, in section 5, some concluding remarks are made.

\section{Random spatially correlated variability}

Irrespective of what approach is used, when predicting the response of structures with variability, some model of the variability is required. Random field theory [12] provides the means for a probabilistic representation of this variability and typically involves expressions for the probability density function together with a model for the spatial variability of the properties, given by a correlation function and correlation length, for a second order homogeneous random field [12]. The most commonly used methods of representing a random field in a mechanical model include the use of series expansions, such as the KL decomposition or the Polynomial Chaos expansion [13]. A random field $H(x, p)$ can be defined as a collection of random variables indexed by a continuous parameter $x \in D$, where $D$ describes the system domain. In other words, for a given 
position $x_{0}, H\left(x_{0}, \theta\right)$ is a random variable, and for a given outcome $\theta, H(x, \theta)$ is a realization of the field.

\subsection{Karhunen-Loève Expansion}

This section presents a brief review of the KL expansion, which is a special case of random field series expansions (e.g. $[19,22,12,40]$ ) where these deterministic spatial functions are orthogonal and derived from the covariance function. A random field $H(x, \theta)$ with a finite, symmetric and positive definite covariance function $C_{H}\left(x_{1}, x_{2}\right)$, defined over a domain $D$, has a spectral decomposition in the form of a series as [13]

$$
H(x)=H_{0}+\sum_{j=1}^{\infty} \sqrt{\lambda_{j}} X_{j} f_{j}(x),
$$

where $H_{0}$ is the random field mean value, $X_{j}$ are uncorrelated random variables and $\lambda_{j}$ and $f_{j}(x)$ are eigenvalues and eigenfunctions, solutions of the Fredholm integral equation of the second kind [13]

$$
\int_{D} C_{H}\left(x_{1}, x_{2}\right) f_{j}\left(x_{1}\right) d x_{1}=\lambda_{j} f_{j}\left(x_{2}\right)
$$

The eigenvalues and eigenfunctions can be ordered in descending order of magnitude of the eigenvalues to truncate the series in Eq. (1) to a finite number of terms $N_{K L}$, chosen by the accuracy of the series in representing the covariance function, rather than the number of random variables [41]. As a rule of thumb, $N_{K L}$ can be chosen such that $\lambda_{N_{K L}} / \lambda_{1}<0.1$, and it depends on the correlation length of the random field. The longer the correlation length the more rapidly the eigenvalues decrease, meaning that fewer terms are needed to accurately represent the series. The random variables $X_{j}$ are obtained from the projection of Eq. (1) on each eigenfunction [13]

$$
X_{j}=\frac{1}{\sqrt{\lambda_{j}}} \int_{D} H(x) f_{j}(x) d x
$$

A random field is Gaussian if the distribution of $H\left(x_{1}\right), H\left(x_{2}\right), \ldots, H\left(x_{n}\right)$ is jointly Gaussian for any $x_{1}, x_{2}, \ldots x_{n}$ within the domain $D$ [40]. If $H(x)$ is a Gaussian random field, $X_{j}$ are always independent, zero mean, unit standard deviation Gaussian random variables, therefore Eq. (3) does not need to be evaluated. If, on the other hand, the random field is not Gaussian, then $X_{j}$ are not independent and have unknown joint PDF. Therefore, it is not possible to use the KL expansion to directly generate a non-Gaussian random field. However, an iterative scheme can be used to overcome this issue [42,43]. It has the advantage of directly using the KL expansion and can simulate both stationary 
and non-stationary random fields as well as strongly non-Gaussian targeted Cumulative Distribution Function (CDF) [44]. Moreover, if the target CDF is approximately Gaussian, only one iteration might be enough to achieve convergence.

In general, the eigenproblem can only be solved numerically and normally involves some procedure for discretizing the random field [19,22,40]. However, for some families of correlation functions and specific geometries, there exist analytical solutions of this integral equation. One such case is the one-dimensional exponentially decaying autocorrelation function

$$
C\left(x_{1}, x_{2}\right)=C\left(x_{1}-x_{2}\right)=\mathrm{e}^{-\left|x_{1}-x_{2}\right| / b},
$$

where $b$ is the correlation length, in the interval $-L / 2 \leq x \leq L / 2$, where $L$ is the length of the domain. The KL expansion for a zero-mean random field is given by [13]

$$
H(x)=\sum_{j=1}^{N_{K L}}\left[X_{1 j} \alpha_{j} \sin \left(w_{1 j} x\right)+X_{2 j} \beta_{j} \cos \left(w_{2 j} x\right)\right]
$$

where $X_{1 j}$ and $X_{2 j}$ are zero-mean, unit standard-deviation, independent random variables with the properties

$$
\left\langle X_{1 j}\right\rangle=\left\langle X_{2 j}\right\rangle=0,\left\langle X_{1 i} X_{2 j}\right\rangle=\delta_{i j}
$$

where $\langle\cdot\rangle$ stands for the mathematical expectation, $\delta_{i j}=1$ for $i=j$ and $\delta_{i j}=0$ for $i \neq$ $j$, and

$$
\alpha_{j}=\sqrt{\frac{\lambda_{1 j}}{\frac{L}{2}-\frac{\sin \left(w_{1 j} L\right)}{2 w_{1 j}}}}, \beta_{j}=\sqrt{\frac{\lambda_{2 j}}{\frac{L}{2}+\frac{\sin \left(w_{2 j} L\right)}{2 w_{2 j}}}}, \lambda_{1 \mathrm{j}}=\frac{2 c}{w_{1 i}^{2}+c^{2}}, \lambda_{2 j}=\frac{2 c}{w_{2 i}^{2}+c^{2}},
$$

where $c=1 / b$ and $w_{1 j}$ and $w_{2 j}$ are the $j^{\text {th }}$ roots of the transcendental equations $c \tan w_{1 j}+w_{1 j}=0$ and $w_{2 j} \tan w_{2 j}-c=0$, respectively. A complete derivation of this solution can be found in the book by Ghanem and Spanos [13]. This analytical solution for the KL expansion is used in the proposed formulation for the sake of simplicity and with no loss of generality. Additionally, it can be shown that correlation length plays a much bigger role than the shape of the correlation function in the statistics of the response [45]. Moreover, a numerical solution to Eq. (2), using a different autocorrelation function, could equally be used.

\section{HFE approximation using the $\mathrm{KL}$ expansion}

A hierarchical finite element formulation is presented in this section. This includes spatial variability of the material or geometrical properties. This formulation not only improves 
the computational cost by decreasing the number of elements necessary to accurately model the structure at higher frequencies, but is also able to be exploited efficiently within a MC framework.

Any displacement variable within a one-dimensional element can be described in terms of $p_{r}$ assumed functions as

$$
u(\xi)=\sum_{r=1}^{p_{r}} g_{r}(\xi) q_{r}=\mathbf{N}(\xi) \mathbf{q}
$$

where $-1 \leq \xi \leq 1$, is the local element coordinate, $\mathbf{N}(\xi)$ is a row-vector containing the functions $g_{r}(\xi)$ and $\mathbf{q}$ is a column vector containing the degrees of freedom $q_{r}$. For twodimensional elements, the displacement field is given by

$$
\mathrm{w}(\xi, \eta)=\sum_{r=1}^{p_{r}} \sum_{s=1}^{p_{s}} g_{r}(\xi) g_{s}(\eta) W_{r s}=\mathbf{N}(\xi, \eta) \mathbf{q}
$$

where $\mathbf{N}(\xi, \eta)$ is the row vector of shape functions, $\mathbf{q}$ is the column vector of degrees of freedom, $\xi$ and $\eta$ are internal element coordinates and $p_{r}$ and $p_{s}$ are the maximum orders of the hierarchical functions. Specific applications are developed for a thin rod in section 3.1, for a thin beam in section 3.2, and for a thin plate in section 3.3. Typically $p_{r}$ and $p_{s}$ are chosen, in homogenous structures, such that the number of half waves in the highest hierarchical function must be greater than the number of half waves in the structural mode whose natural frequency is being calculated [29,46]. However, because the element properties are not constant along the element, the choice of the order of the hierarchical function also has to take into account the correlation length of the random field. From Eq. (5), $w_{1 j}$ and $w_{2 j}$ are the spatial frequency of each term in the expansion. The spatial frequency increases with the increasing order of the expansion such that $j=N_{K L}$ gives the maximum spatial frequency contained in the series. In general, a relation can be found between the KL series and a spectral representation [41] as is shown, for example, in Figure 1 . It presents the largest eigenvalues $\lambda_{j}$ as a function of the spatial frequency $w_{j}$ normalized by the element size $L$. Each eigenvalue $\lambda_{j}$ is normalized by the largest eigenvalue $\lambda_{1}$. The number of eigenvalues is truncated to $N_{K L}$. Note that the eigenvalues decrease more rapidly for longer correlation lengths. Therefore, fewer terms are necessary for convergence of the series in Eq. (5) when compared to shorter correlation lengths to achieve the same level of accuracy, given by the ratio $\lambda_{j} / \lambda_{1}$. Hence, a higher spatial frequency content, i.e. $w_{1 j}$ and $w_{2 j}$, is expected in random fields with shorter correlation length.

In a standard Finite Element implementation, the random field has to be discretized in the stochastic domain, typically done by using the KL expansion, and in the geometrical domain, at the element level. The size of the finite elements should be such that it can capture the essential features of the spatial variability in addition to the number of the 
half-waves in the structural modes. Typically, a rule-of-thumb stablishing the ratio of the length of the finite elements and the correlation length can be used for most correlation function families (e.g. [47,48]). The same principle applies for the HFE method, in which the order of the hierarchical function has to capture both number of half-waves in the structural modes and the random fluctuations due to the spatial correlation. A first approach is to ensure that the number of half waves in the highest hierarchical function must also be greater than the number of half waves in the highest term of the KL series. A convergence study involving several families and of hierarchical functions, correlation families and correlation lengths for 1D and 2D structures is necessary to address to issue properly, and is beyond the scope of this paper.

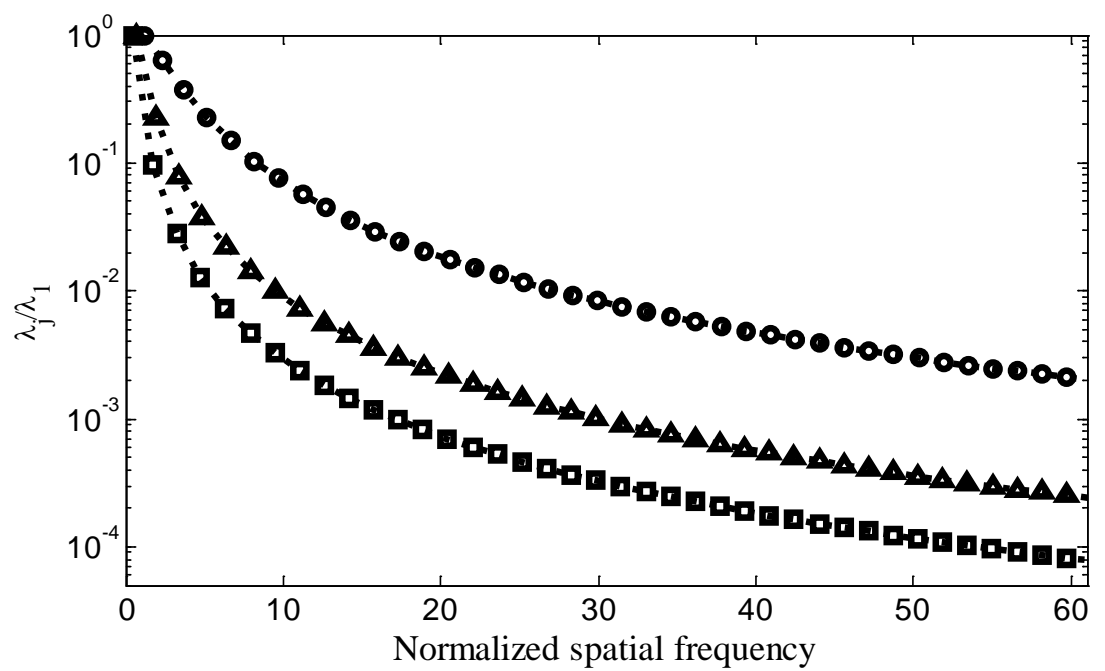

Figure 1. Normalized spectral representation of the KL expansion for three correlation lengths: $b=2 L$ (square dotted line), $b=0.8 L$ (triangle dotted line), and $b=0.2 L$ (circle dotted line).

The formulation of the element matrices using the KL expansion allows them to be rewritten as a sum of the nominal stiffness and mass matrices calculated with the mean properties and a term accounting for the spatially varying properties. In general, the stiffness and mass matrices are given respectively by

$$
\begin{array}{r}
\mathbf{K}_{e}=\mathbf{K}_{e 0}+\Delta \mathbf{K}_{e}, \\
\mathbf{M}_{e}=\mathbf{M}_{e 0}+\Delta \mathbf{M}_{e},
\end{array}
$$

in which $\mathbf{K}_{\boldsymbol{e} \mathbf{0}}$ and $\mathbf{M}_{\boldsymbol{e} \mathbf{0}}$ are the matrices obtained from the standard HFE approach, using mean homogeneous properties, and $\Delta \mathbf{K}_{\boldsymbol{e}}$ and $\Delta \mathbf{M}_{\boldsymbol{e}}$ are matrices obtained by using the spatial functions of the KL expansion in order to represent the random field. In this way, the element integration is performed for each one of the terms individually, so that 


$$
\begin{aligned}
\Delta \mathbf{K}_{\boldsymbol{e}} & =\sum_{j=1}^{N_{K L}} X_{j} \Delta \mathbf{K}_{j e} \\
\Delta \mathbf{M}_{\boldsymbol{e}} & =\sum_{j=1}^{N_{K L}} Y_{j} \Delta \mathbf{M}_{j e}
\end{aligned}
$$

in which $X_{j}$ and $Y_{j}$ are zero-mean, unit standard deviation and independent random variables. Dividing the integration into separate integrals for each KL term can increase the overall computational cost of including the spatially varying properties. However, within a MC framework this integration step is only performed once and can reduce significantly the total sampling time.

Typical applications involve built-up structures and assemblies of plates and beams $[31,49]$. Wave-based approaches can be used to model such assemblies but they typically cannot take spatially correlated uncertainty into account [50,51], unless it is assumed that the properties are slowly varying [52,53]. Usually, the vibration analysis of a plate using the standard $h$-version FE method would require many elements while the HFE requires only one element, producing smaller mass and stiffness matrices. However, unlike the case for which the element properties are constant, the element integration step is costly when compared to the solution of the system itself. By using Eqs. (12) and (13), the element integration process is performed only once and the random terms $\Delta \mathbf{K}_{\boldsymbol{e}}$ and $\Delta \mathbf{M}_{\boldsymbol{e}}$ can be sampled in a MC scheme by sampling $X_{j}$ and $Y_{j}$, with no need to repeat the calculation of the element matrices. This approach is particularly beneficial in conjunction with the KL expansion, which provides the smallest number of terms in the series to represent the random field to a given accuracy [13,54]. Other sampling approaches, such as line-sampling [5,55], could take additional advantage of this formulation to reduce the number of samples needed to accurately calculate the response statistics. Details of the formulation will be shown for the examples of rod, beam and plate elements in the following sections.

\subsection{Axial vibration of rods}

In this formulation, the axial vibration of a thin rod and an element formulation with two nodes is considered, each node with one degree of freedom. Moreover, the spatial variability of the material or geometrical properties is assumed to be homogeneous, i.e. it has a constant mean value. The displacement field along the element is given by Eq. (8) and the first two shape functions are taken to be $g_{1}(\xi)=\frac{1}{2}(1-\xi), g_{2}=\frac{1}{2}(1+\xi)$. The most commonly used hierarchical functions $g_{r}(\xi)$, are derived from the Legendre polynomials [1]. For the rod element they are given, for $r>2$, by 


$$
g_{r}(\xi)=\sum_{n=0}^{(r-1) / 2} \frac{(-1)^{n}}{2^{n} n !} \frac{(2 r-2 n-5) ! !}{(r-2 n-1) !} \xi^{r-2 n-1}
$$

where $m ! !=m(m-2) \ldots(2$ or 1$), 0 ! !=1,(-1) ! !=1$ and the limit $(r-1) / 2$ of the sum stands for its integer part. This ensures that the contribution of the terms at the nodes $\xi= \pm 1$ is zero, therefore there is no increase in the number of nodes.

Assuming spatial variability in the material and geometrical properties such that the longitudinal stiffness is given by $E(x) A(x)=E A(x)$ and the mass per unit length is given by $\rho(x) A(x)=\rho A(x)$, the element matrices can be written as

$$
\begin{aligned}
& \mathbf{K}_{e}=\frac{1}{a} \int_{-1}^{1} E A(\xi) \mathbf{N}(\xi)^{\prime T} \mathbf{N}(\xi)^{\prime} d \xi \\
& \mathbf{M}_{e}=a \int_{-1}^{1} \rho A(\xi) \mathbf{N}(\xi)^{T} \mathbf{N}(\xi) d \xi
\end{aligned}
$$

where $a=L / 2, L$ being the element size, ' stands for the first spatial derivative, the row matrix $\mathbf{N}(\xi)$ contains the functions $g_{r}(\xi)$ and the material and geometrical properties are written in terms of the local coordinate $\xi$. Note that this assumption combines variability in geometrical and material properties and simplifies the overall KL representation. Moreover, the force vector for the element can be calculated as $\mathbf{f}_{\mathbf{e}}=a \int_{-1}^{1} p_{x} \mathbf{N}(\xi) d \xi$, where $p_{x}$ is the applied load per unit length. For point excitation at $x_{e}$, then $p_{x}=f_{x} \delta(x-$ $x_{e}$ ), where $\delta(x)$ is the Dirac delta function, then $\mathbf{f}_{\mathbf{e}}=a f_{x} \mathbf{N}\left(x_{e}\right)$.

It is assumed that the spatially varying properties are described in the same manner as in Eq. (5), i.e.

$$
\begin{aligned}
& E A(x)=E A_{0}\left[1+\sigma_{E A} \sum_{j=1}^{N_{K L}}\left(X_{1 j} \alpha_{j} \sin \left(w_{1 j} x\right)+X_{2 j} \beta_{j} \cos \left(w_{2 j} x\right)\right)\right] \\
& \rho A(x)=\rho A_{0}\left[1+\sigma_{\rho A} \sum_{j=1}^{N_{K L}}\left(Y_{1 j} \gamma_{j} \sin \left(w_{3 j} x\right)+Y_{2 j} \epsilon_{j} \cos \left(w_{4 j} x\right)\right)\right] .
\end{aligned}
$$

where $E A_{0}$ and $\rho A_{0}$ are the mean values for the axial stiffness and the mass per unit length and $\sigma_{E A}$ and $\sigma_{\rho A}$ are the dispersion terms, quantifying the influence of the random field on the mean nominal value, the coefficients $\alpha_{j}, \beta_{j}, \gamma_{j}$ and $\epsilon_{j}$ are the coefficients of the KL series, Eq. (7) and $X_{1 j}, X_{2 j}, Y_{1 j}$ and $Y_{2 j}$ are zero-mean, unit standard-deviation, independent random variables. The element stiffness matrix is given by Eq. (10), where $\Delta \mathbf{K}_{e}=\Delta \mathbf{K}_{e 1}+\Delta \mathbf{K}_{e 2}$, with 


$$
\Delta \mathbf{K}_{e 1}=\frac{\sigma_{E A}}{a} \sum_{j=1}^{N_{K L}} X_{1 j} \Delta \mathbf{K}_{j e 1}, \quad \Delta \mathbf{K}_{e 2}=\frac{\sigma_{E A}}{a} \sum_{j=1}^{N_{K L}} X_{2 j} \Delta \mathbf{K}_{j e 2},
$$

and

$$
\begin{aligned}
& \mathbf{\Delta K}_{j e 1}=\alpha_{j} \int_{-1}^{1} \sin \left(\frac{2 w_{1 j}}{L} \xi\right)\left\lfloor\mathbf{N}(\xi)^{\prime}\right\rfloor^{T}\left\lfloor\mathbf{N}(\xi)^{\prime}\right\rfloor d \xi, \\
& \Delta \mathbf{K}_{j e 2}=\beta_{j} \int_{-1}^{1} \cos \left(\frac{2 w_{2 j}}{L} \xi\right)\left\lfloor\mathbf{N}(\xi)^{\prime}\right\rfloor^{T}\left\lfloor\mathbf{N}(\xi)^{\prime}\right\rfloor d \xi .
\end{aligned}
$$

Therefore, expressions for the $(m, n)$-th element of each matrix can be written as

$$
\begin{gathered}
\Delta \mathrm{K}_{j e 1}^{(m, n)}=\alpha_{j} \int_{-1}^{1} g_{m}(\xi)^{\prime} g_{n}(\xi)^{\prime} \sin \left(\frac{w_{1 j} L}{L} \xi\right) d \xi, \\
\Delta \mathrm{K}_{j e 2}^{(m, n)}=\beta_{j} \int_{-1}^{1} g_{m}(\xi)^{\prime} g_{n}(\xi)^{\prime} \cos \left(\frac{2 w_{2 j}}{L} \xi\right) d \xi,
\end{gathered}
$$

where $m, n=1, \ldots, r$. The element mass matrix is given by Eq. (11), where $\Delta \mathbf{M}_{e}=$ $\Delta \mathbf{M}_{e 1}+\Delta \mathbf{M}_{e 2}$, with

$$
\Delta \mathbf{M}_{e 1}=a \sigma_{\rho A} \sum_{j=1}^{N_{K L}} Y_{1 j} \Delta \mathbf{M}_{j e 1}, \quad \Delta \mathbf{M}_{e 2}=a \sigma_{\rho A} \sum_{j=1}^{N_{K L}} Y_{2 j} \Delta \mathbf{M}_{j e 2} .
$$

and

$$
\begin{aligned}
& \mathbf{\Delta M}_{j e 1}=\gamma_{j} \int_{-1}^{1} \sin \left(\frac{2 w_{3 j}}{L} \xi\right)\lfloor\mathbf{N}(\xi)\rfloor^{T}\lfloor\mathbf{N}(\xi)\rfloor d \xi \\
& \mathbf{M}_{j e 2}=\epsilon_{j} \int_{-1}^{1} \cos \left(\frac{2 w_{4 j}}{L} \xi\right)\lfloor\mathbf{N}(\xi)\rfloor^{T}\lfloor\mathbf{N}(\xi)\rfloor d \xi
\end{aligned}
$$

The $(m, n)$-th elements of theses matrices can also written as

$$
\begin{aligned}
& \Delta \mathrm{M}_{j e 1}^{(m, n)}=\gamma_{j} \int_{-1}^{1} g_{m}(\xi) g_{n}(\xi) \sin \left(\frac{2 w_{3 j}}{L} \xi\right) d \xi, \\
& \Delta \mathrm{M}_{j e 2}^{(m, n)}=\epsilon_{j} \int_{-1}^{1} g_{m}(\xi) g_{n}(\xi) \cos \left(\frac{2 w_{4 j}}{L} \xi\right) d \xi .
\end{aligned}
$$


The terms, $\Delta \mathrm{K}_{j e 1}^{(m, n)}, \Delta \mathrm{K}_{j e 1}^{(m, n)}, \Delta \mathrm{M}_{j e 1}^{(m, n)}$ and $\Delta \mathrm{M}_{j e 2}^{(m, n)}$, lead to symmetric matrices and need to be calculated only once, which reduces the overall computational cost of calculating the statistics of the response in a MC framework. Numerical evaluation can be done by using a quadrature rule. Besides, this approach ensures that the inclusion of more hierarchical functions, for higher order approximations, does not affect the elements of the matrices that have already been calculated, therefore increasing the overall numerical efficiency of the method [1].

\subsection{Bending vibration of beams}

In this section the hierarchical finite element including material variability in the element formulation is developed for beams in flexural vibration using Euler-Bernoulli beam theory. The formulation follows the same procedure as that for the rod element, but using a different set of hierarchical functions. It also produces smaller matrices than the usual FE approach would, with no increase in the number of the degrees of freedom, only enriching the internal displacement field. The element considered has two nodes with two degrees of freedom at each node. Also, the spatial variability of the material or geometrical properties are assumed to have constant mean values. The flexural displacement along the element can be described in terms of $p$ assumed functions, as shown in Eq. (8). The first four shape functions are the usual finite element shape functions $g_{1}(\xi)=\frac{1}{4}\left(2-3 \xi+\xi^{2}\right), g_{2}=\frac{1}{4}\left(1-\xi-\xi^{2}+x^{3}\right), g_{3}=\frac{1}{4}\left(2+3 \xi-x^{3}\right)$, $g_{4}=\frac{1}{4}\left(-1-\xi+\xi^{2}+x^{3}\right)$, and the hierarchical functions

$$
g_{r}(\xi)=\sum_{n=0}^{(r-1) / 2} \frac{(-1)^{n}}{2^{n} n !} \frac{(2 r-2 n-7) ! !}{(r-2 n-1) !} \xi^{r-2 n-1}
$$

for $r>4$ are also derived from the Legendre polynomials, but with a slight modification. Spatial variability in the flexural stiffness and mass per unit length $E(x) I_{y y}(x)=$ $E I_{y y}(x)$ and $\rho(x) A(x)=\rho A(x)$, are included in the stiffness and mass matrices by

$$
\begin{gathered}
\mathbf{K}_{e}=\frac{1}{a^{3}} \int_{-1}^{1} E I_{y y}(\xi) \mathbf{N}(\xi)^{\prime \prime T} \mathbf{N}(\xi)^{\prime \prime} d \xi, \\
\mathbf{M}_{e}=a \int_{-1}^{1} \rho A(\xi) \mathbf{N}(\xi)^{T} \mathbf{N}(\xi) d \xi
\end{gathered}
$$

where $a=L / 2, L$ being the element size, and " stands for the second spatial derivative. Moreover, the force vector can be calculated as $\mathbf{f}_{\mathbf{e}}=a \int_{-1}^{1} p_{x} \mathbf{N}(\xi) d \xi$. For point excitation, then $\mathbf{f}_{\mathbf{e}}=a f_{x} \mathbf{N}\left(x_{e}\right)$. The KL expansion, Eq. (5), is used for modelling the spatially varying properties, i.e. 


$$
\begin{gathered}
E I_{y y}(x)=E I_{y y_{0}}\left[1+\sigma_{E I} \sum_{j=1}^{N_{K L}}\left(X_{1 j} \alpha_{j} \sin \left(w_{1 j} x\right)+X_{2 j} \beta_{j} \cos \left(w_{2 j} x\right)\right)\right] \\
\rho A(x)=\rho A_{0}\left[1+\sigma_{\rho A} \sum_{j=1}^{N_{K L}}\left(Y_{1 j} \gamma_{j} \sin \left(w_{3 j} x\right)+Y_{2 j} \epsilon_{j} \cos \left(w_{4 j} x\right)\right)\right] .
\end{gathered}
$$

The element stiffness matrix is given by Eq. (10) where $\Delta \mathbf{K}_{e}=\Delta \mathbf{K}_{e 1}+\Delta \mathbf{K}_{e 2}$, with

$$
\Delta \mathbf{K}_{e 1}=\frac{\sigma_{E I}}{a^{3}} \sum_{j=1}^{N_{K L}} X_{1 j} \Delta \mathbf{K}_{j e 1}, \quad \Delta \mathbf{K}_{e 2}=\frac{\sigma_{E I}}{a^{3}} \sum_{j=1}^{N_{K L}} X_{2 j} \Delta \mathbf{K}_{j e 2}
$$

and

$$
\begin{aligned}
& \Delta \mathbf{K}_{j e 1}=\alpha_{j} \int_{-1}^{1} \sin \left(\frac{2 w_{1}}{L} \xi\right) \mathbf{N}(\xi)^{\prime \prime T} \mathbf{N}(\xi)^{\prime \prime} d \xi \\
& \Delta \mathbf{K}_{j e 2}=\beta_{j} \int_{-1}^{1} \cos \left(\frac{2 w_{2}}{L} \xi\right) \mathbf{N}(\xi)^{\prime \prime T} \mathbf{N}(\xi)^{\prime \prime} d \xi
\end{aligned}
$$

The mass matrix is given by Eq. (11), where $\Delta \mathbf{M}_{e}=\Delta \mathbf{M}_{e 1}+\Delta \mathbf{M}_{e 2}$, with

$$
\Delta \mathbf{M}_{e 1}=2 a \sigma_{\rho A} \sum_{j=1}^{N_{K L}} Y_{1 j} \Delta \mathbf{M}_{j e 1}, \quad \Delta \mathbf{M}_{e 2}=2 a \sigma_{\rho A} \sum_{j=1}^{N_{K L}} Y_{2 j} \Delta \mathbf{M}_{j e 2}
$$

and

$$
\begin{aligned}
\Delta \mathbf{M}_{j e 1} & =\gamma_{j} \int_{-1}^{1} \sin \left(\frac{2 w_{3 \mathrm{j}}}{L} \xi\right) \mathbf{N}(\xi)^{T} \mathbf{N}(\xi) d \xi \\
\Delta \mathbf{M}_{j e 2} & =\epsilon_{j} \int_{-1}^{1} \cos \left(\frac{2 w_{4 \mathrm{j}}}{L} \xi\right) \mathbf{N}(\xi)^{T} \mathbf{N}(\xi) d \xi .
\end{aligned}
$$

Expressions for the $m^{\text {th }}$ row and $n^{\text {th }}$ column element of each matrix are given by Eqs. (21) and (22) for the stiffness matrices and Eq. (27) and (28) for the mass matrices. This approach also ensures that the inclusion of more hierarchical functions, for higher order approximations, does not affect the elements of the matrices that were already calculated. Besides, they are only calculated once, which reduces the computation cost in a MC sampling scheme, and can be numerically evaluated by using a quadrature rule. 


\subsection{Flexural vibration of plates}

Two-dimensional spatial variability can also be included by using the HFE method $[29,36,56,57]$. In this section, a two-dimensional thin, rectangular and isotropic plate element is developed, again assuming that the spatial variability of the material and geometrical properties have constant mean values. A conforming rectangular element is used which has four degrees of freedom per node and four nodes, one at each corner, enriched with the most commonly used trigonometric type hierarchical functions [1]

$$
g_{r, s}(\xi)=\frac{1}{4}\left(1-\xi^{2}\right) \sin \left[\frac{\pi}{2}(r-4)(1+\xi)\right]
$$

for $r, s>4$. The first 16 degrees of freedom are the same as the conforming FE [1]. It is recommended that trigonometric functions are used instead of polynomials for improved accuracy at higher frequencies. Two-dimensional elements are more sensitive to this choice due to rounding errors. The first four functions are the standard shape functions for a thin plate in bending [1].

Assuming two-dimensional spatial variability in the material properties, the element matrices are of size $p^{2}$ by $p^{2}$ and can be written as

$$
\begin{aligned}
& \mathbf{K}_{e}=\frac{a b}{12} \int_{-1}^{1} \int_{-1}^{1} \mathbf{B}(\xi, \eta)^{T} \mathbf{D}(\xi, \eta) \mathbf{B}(\xi, \eta) d \xi d \eta \\
& \mathbf{M}_{e}=a b \int_{-1}^{1} \int_{-1}^{1} \rho h(\xi, \eta) \mathbf{N}(\xi, \eta)^{T} \mathbf{N}(\xi, \eta) d \xi d \eta
\end{aligned}
$$

where $a=L_{x} / 2, b=L_{y} / 2$ and $h$ is the plate thickness. From the stress-strain relations of an isotropic plate,

$$
\mathbf{D}(\xi, \eta)=E(\xi, \eta) h^{3}(\xi, \eta)\left[\begin{array}{ccc}
\frac{1}{\left(1-v^{2}\right)} & \frac{v}{\left(1-v^{2}\right)} & 0 \\
\frac{v}{\left(1-v^{2}\right)} & \frac{1}{\left(1-v^{2}\right)} & 0 \\
0 & 0 & \frac{1}{2(1+v)}
\end{array}\right]
$$

in which the Poisson's ratio $v$ is assumed homogeneous, and 


$$
\mathbf{B}(\xi, \eta)=\left[\begin{array}{c}
\frac{1}{a^{2}} \frac{\partial^{2}}{\partial \xi^{2}} \\
\frac{1}{b^{2}} \frac{\partial^{2}}{\partial \eta^{2}} \\
\frac{2}{a b} \frac{\partial^{2}}{\partial \xi \partial \eta}
\end{array}\right] \mathbf{N}(\xi, \eta)
$$

Moreover, the force vector can be calculated as $\mathbf{f}_{\mathbf{e}}=a b \int_{-1}^{1} \int_{-1}^{1} p_{x y} \mathbf{N}(\xi, \eta) d \xi d \eta$, where $p_{x y}$ is the force per unit area. For point excitation $p_{x y}=F \delta\left(x-L_{1 x}\right) \delta\left(y-L_{1 y}\right)$ applied at $\left(L_{1 x}, L_{1 y}\right)$, then $\mathbf{f}_{\mathbf{e}}=a b F \mathbf{N}\left(x_{e}, y_{e}\right)$. The two-dimensional spatially varying properties can be written in terms of the analytical solution of the KL expansion, Eq. (5), by choosing a separable autocorrelation function in the rectangular domain $-L_{x} / 2 \leq x \leq L_{x} / 2$ and $-L_{y} / 2 \leq y \leq L_{y} / 2$ [12], e.g.

$$
C\left(x_{1}-x_{2}, y_{1}-y_{2}\right)=C\left(x_{1}-x_{2}\right) C\left(y_{1}-y_{2}\right)=\mathrm{e}^{-\left|x_{1}-x_{2}\right| / b_{x}} \mathrm{e}^{-\left|y_{1}-y_{2}\right| / b_{y}},
$$

where $b_{x}$ and $b_{y}$ are the correlation lengths in the $x$ and $y$ direction, respectively. Note that $b_{x}$ and $b_{y}$ can assume different values so that the correlation is different for each $x$ and $y$ direction. This is required for the case of, for example, fibre reinforced composites $[8,9]$. This property ensures that the problem separates into two independent onedimensional problems, so that $f_{r}(x, y)=f_{i}(x) f_{j}(y)$ and $\lambda_{r}=\lambda_{i} \lambda_{j}$. Note that the eigenvalues $\lambda_{-}(\mathrm{i}, \mathrm{j})$ can be directly calculated in decreasing order, but the $\lambda_{-} \mathrm{r}$ have to be sorted in that way such that the expansion can be truncated according to the rule of thumb $\lambda \_r / \lambda \_1<0.1$. The choice of notation for the eigenfunction, in this case, is then changed for practical computational implementation. Therefore

$$
\begin{gathered}
E h^{3}(x, y)=E_{0} h_{0}^{3}\left[1+\sigma_{E} \sum_{r=1}^{N_{K L}} \sqrt{\lambda_{1 r}} X_{r} f_{1 x r}(x) f_{1 y r}(y)\right], \\
\rho h(x)=\rho_{0} h_{0}\left[1+\sigma_{\rho} \sum_{r=1}^{N_{K L}} \sqrt{\lambda_{2 r}} Y_{r} f_{2 x r}(x) f_{2 y r}(y)\right],
\end{gathered}
$$

where $E_{0}, \rho_{0}$ and $h_{0}$ are the mean values of Young's modulus, mass density and thickness, $\sigma_{E}$ and $\sigma_{\rho}$ are the dispersion parameters, $X_{r}$ and $Y_{r}$ are zero-mean, unit standard-deviation independent random variables, $f_{1 x r}(x), f_{1 x y}(y), f_{2 x r}(x)$ and $f_{1 x y}(y)$ are the eigenfunctions from the analytical expression of the KL expansion, Eq. (5), $\lambda_{1 r}$ and $\lambda_{2 r}$ are the corresponding eigenvalues and $N_{K L}$ is the number of terms used in the expansion. The element matrices can be rewritten as a sum of the usual stiffness and mass matrices 
found with homogeneous properties and a term accounting for the spatially varying properties, i.e. $\mathbf{K}_{e}=\mathbf{K}_{e 0}+\Delta \mathbf{K}_{e}$ with

$$
\Delta \mathbf{K}_{e}=\sigma_{E} E_{0} h_{0}^{3} \frac{a b}{12} \sum_{r=1}^{N_{K L}} \sqrt{\lambda_{1 r}} X_{r} \Delta \mathbf{K}_{r e}
$$

and

$$
\Delta \mathbf{K}_{r e}=\int_{-1}^{1} \int_{-1}^{1} f_{1 x r}\left(\frac{2 \xi}{L_{x}}\right) f_{1 y r}\left(\frac{2 \eta}{L_{y}}\right) \mathbf{B}(\xi, \eta)^{T} \widetilde{\mathbf{D}} \mathbf{B}(\xi, \eta) d \xi d \eta,
$$

with

$$
\widetilde{\mathbf{D}}=\left[\begin{array}{ccc}
\frac{1}{\left(1-v^{2}\right)} & \frac{v}{\left(1-v^{2}\right)} & 0 \\
\frac{v}{\left(1-v^{2}\right)} & \frac{1}{\left(1-v^{2}\right)} & 0 \\
0 & 0 & \frac{1}{2(1+v)}
\end{array}\right]
$$

and $\mathbf{M}_{e}=\mathbf{M}_{e 0}+\Delta \mathbf{M}_{e}$, with

$$
\Delta \mathbf{M}_{e}=\sigma_{\rho} \rho h_{0} a b \sum_{r=1}^{N_{K L}} \sqrt{\lambda_{2 r}} Y_{r} \Delta \mathbf{M}_{r e}
$$

and

$$
\Delta \mathbf{M}_{r e}=\int_{-1}^{1} \int_{-1}^{1} f_{2 x r}\left(\frac{2 \xi}{L_{x}}\right) f_{2 y r}\left(\frac{2 \eta}{L_{y}}\right) \mathbf{N}(\xi, \eta)^{T} \mathbf{N}(\xi, \eta) d \xi d \eta
$$

The integral terms $\Delta \mathbf{K}_{j e}$ and $\Delta \mathbf{M}_{j e}$, are only calculated once and can be numerically evaluated by using a quadrature rule, reducing the overall computation cost a MC sampling scheme. Unlike the rod and beam formulation, these terms require a double integration because the plate element is two-dimensional.

\section{Numerical examples}

Numerical examples are presented in this section to compare results obtained with the stochastic HFE approximation and FE prediction. MC simulations were performed using $N_{s}=1,000$ samples, for both HFE and stochastic FE approaches, sufficient for adequate convergence of the mean and standard deviation of the forced response. The FE method is used as a benchmark solution. For the rod and beam cases, 


$$
E(x)=H(x)
$$

where $H(x)$ is a Gamma distributed homogeneous random field with correlation function given by Eq. (4) and therefore an analytical expression for the KL expansion can be used, as given in Eq. (5). The Gamma distribution is the best stochastic model for the Young's modulus according to a Maximum Entropy criterion [58], based on the available information about the random variable or process. The mean value is $E_{0}$, which is the nominal model chosen for the Young's modulus, the value of $E$ is always positive and non-zero, i.e. $0<E(x)<+\infty$, and also $\langle\ln E(x)\rangle<+\infty$. The latter assumption ensures that the second order moment statistics of the response are finite. It can be shown that the use of distributions not meeting these criteria, like the Gaussian or exponential distribution, for instance, is not physically sound because it means that the expected value of the deformation energy is undefined [58,59]. The Gamma distribution is given by

$$
f(x)=x^{a_{0}-1} \frac{\exp \left(-\frac{x}{b_{0}}\right)}{b_{0}^{a_{0}} \Gamma\left(a_{0}\right)}, x>0,
$$

where $\Gamma(x)=\int_{0}^{\infty} t^{x-1} e^{-t} d t, x>0$ is the Gamma function and the parameters of the distribution are given by $a_{0}=1 / \delta_{E}^{2}$ and $b_{0}=E_{0} \delta_{E}^{2}$, where $\delta_{E}$ is the dispersion parameter. Equation (5) is used to simulate the random field in which $X_{1 j}$ and $X_{2 j}$ are firstly generated using a Gamma independent random variables, generated using a Latin Hypercube sampling scheme [39,60]. Only one iteration is needed to meet the criteria proposed by Phoon et al. [42,43] because the given parameters of the Gamma distribution are such that it is very close to a Gaussian distribution.

For the plate, variability of the Young's modulus is also considered assuming a Gamma homogeneous random field $H(x, y)$, but with a separable correlation function, so that the analytical expression for the KL expansion can also be used.

Additionally, the rod, beam and plate properties are assumed to be $E_{0}=210 \mathrm{GPa}$ and $\rho=7800 \mathrm{~kg} / \mathrm{m}^{3}$. Structural damping is included by allowing the Young's modulus $E_{0}=E_{0}(1+i \tilde{\eta})$ to be complex, with a hysteretic loss factor $\tilde{\eta}=10^{-3}$.

The mid-point method [18] is used to discretize the random field along with the KL expansion in the FE elements. It approximates the random field by choosing a constant value for each element based on the KL expansion at its centroid. This choice of element size ensures an accurate representation of the random field in the $x$ and $y$ direction, for a given correlation length $[12,54,61]$.

\subsection{Rod}

In this case, a rod with a cross-sectional area $A=1 \times 3 \mathrm{~cm}^{2}$, total length $L=4 \mathrm{~m}$, with free-free boundary conditions is chosen. The frequency band under analysis is up to $3.5 \mathrm{kHz}$, discretised in steps of $1 \mathrm{~Hz}$, to include the first five natural frequencies (plus of 
course the rigid body mode). The excitation point is at $x=L_{1}=0.2 L$. Results are compared with a standard FE approach using 100 elements.

Three different cases of the random field of the Young's modulus are considered, by changing the dispersion parameter $\delta_{E}$ and the correlation length $b$. Values considered are $\delta_{E}=0.1, b=2 L ; \delta_{E}=0.1, b=0.8 L$ and $\delta_{E}=0.15, b=0.2 L$, the latter having a much shorter correlation length with a larger standard deviation. For each of these cases, one sample was generated, as shown in Figure 2, using $N_{K L}=5$, i.e. ten terms in the series expansion Eq. (5), which is enough to meet the convergence criterion $\lambda_{k} / \lambda_{1}<0.1$. From Figure 2, it is possible to observe that for the longer correlation lengths, the spatial distribution has very distinguishable components of long length scale with relatively small fluctuations. This is because, from Eq. (5), the sine and cosine terms with longer wavelengths have relatively much more importance than the shorter ones. That relative importance decreases for shorter correlation lengths and increases the scale of the fluctuations along the length of the structure.

These samples were used as deterministic cases for calculating the input mobility at $0.2 \mathrm{~L}$, i.e. the Frequency Response Function (FRF) given by the ratio of the velocity and a point force at the excitation location, and are shown in Figure 3. They are calculated by the direct inversion of the dynamic stiffness matrix in the HFE case and modal summation in the FE model. Differences in the results in Figure 3(c) are due to the neglect of higher order FE modes. The maximum order of the hierarchical functions was chosen to be $p=$ 14, using Eq. (8), which leads to a total of 14 degrees of freedom while the FE model has 101 in total.

Overall, the HFE and FE results are in very good agreement for all of the cases. This is important for the further calculation of the response statistics. Natural frequencies calculated using the HFE method are less than $0.1 \%$ different to those from the FE model. Figure 4 presents the mean value and $5^{\text {th }}$ and $95^{\text {th }}$ percentile of the input mobility and Figure 5 shows the PDFs of the $4^{\text {th }}$ natural frequency, a typical result, both obtained from the MC sampling scheme for each pair of $b$ and $\delta_{E}$. The PDFs are normalized by the mean value of the distribution calculated from the HFE and FE models, respectively, and is also the case for the beam and plate cases. The same random field samples were used to calculate the results from both HFE and FE methods and the non-smoothness in the PDFs is due to the size of these samples. 


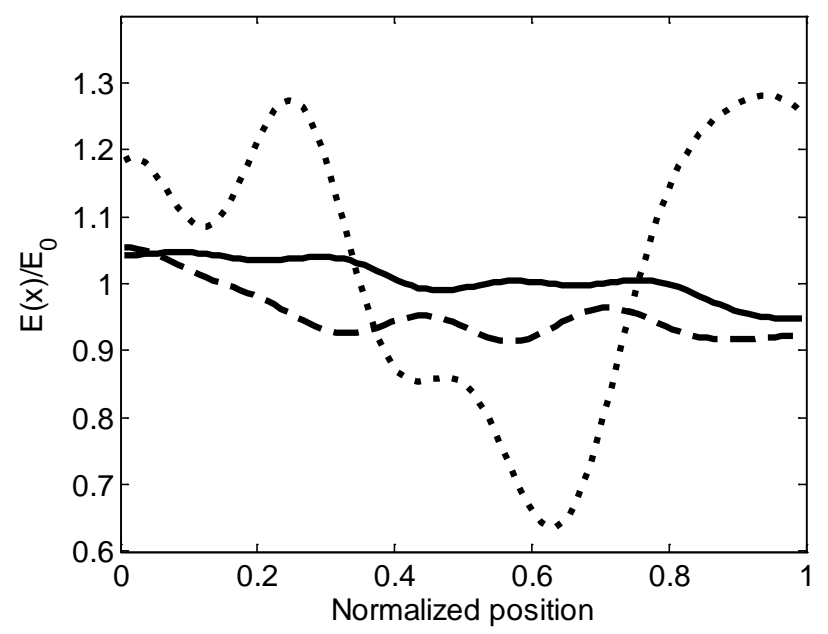

Figure 2. Samples of normalized Young's modulus as a function of the normalized position used for the rod and beam elements, for the cases dispersion parameter $\delta_{E}$ and correlation length $b$ : $\delta_{E}=0.1, b=2 L$ (solid line) $\delta_{E}=0.1, b=0.8 L$ (dashed line); and $\delta_{E}=0.15, b=0.2 L$ (dotted line). 
(a)
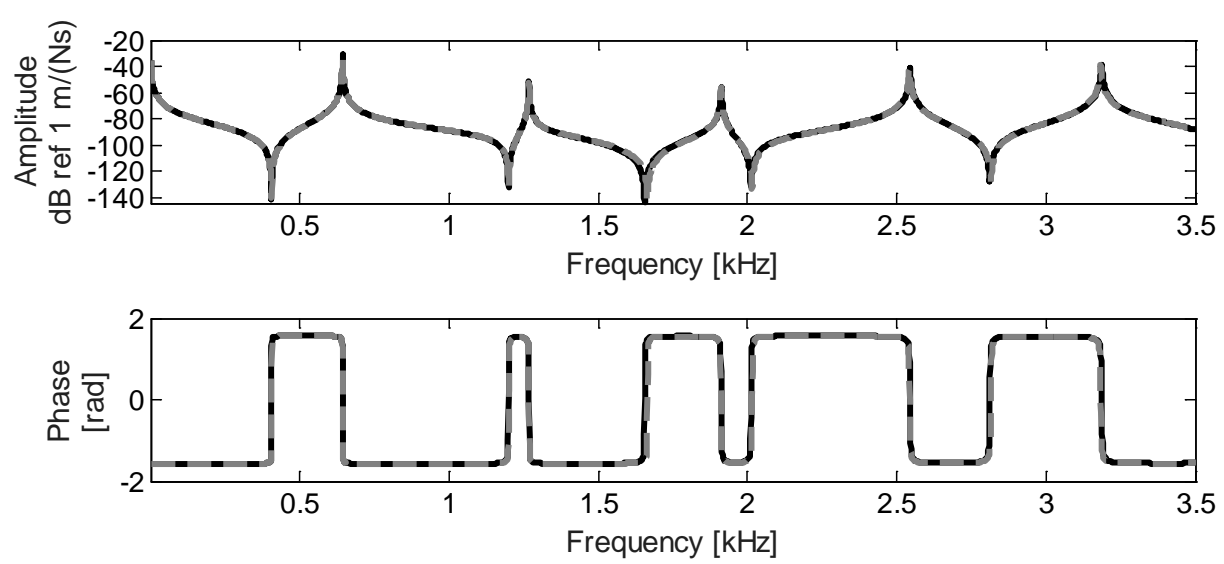

(b)
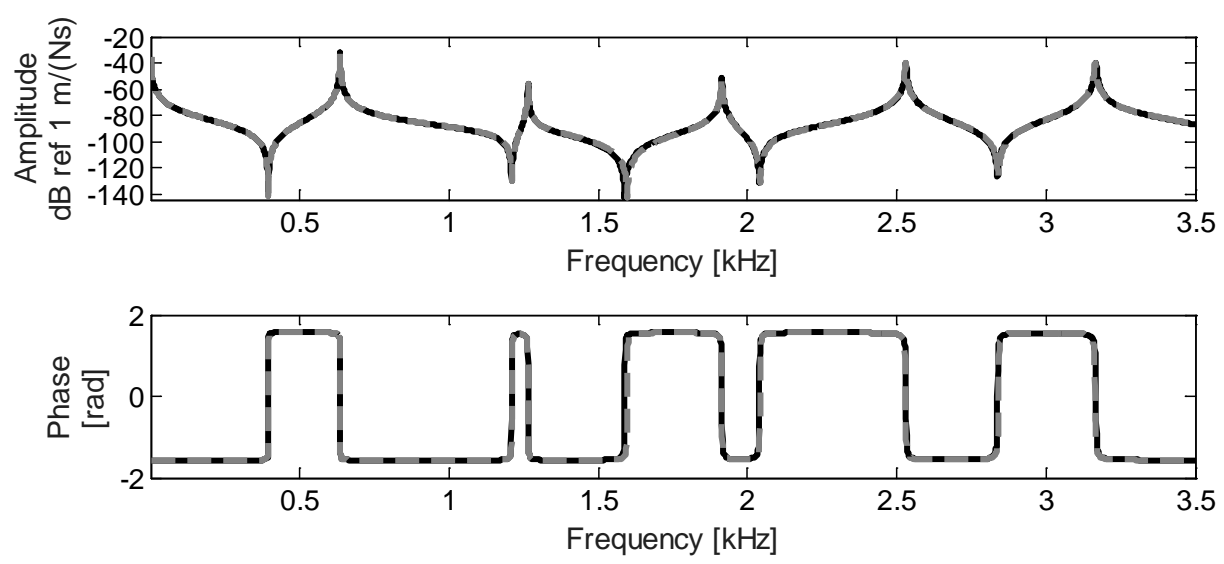

(c)
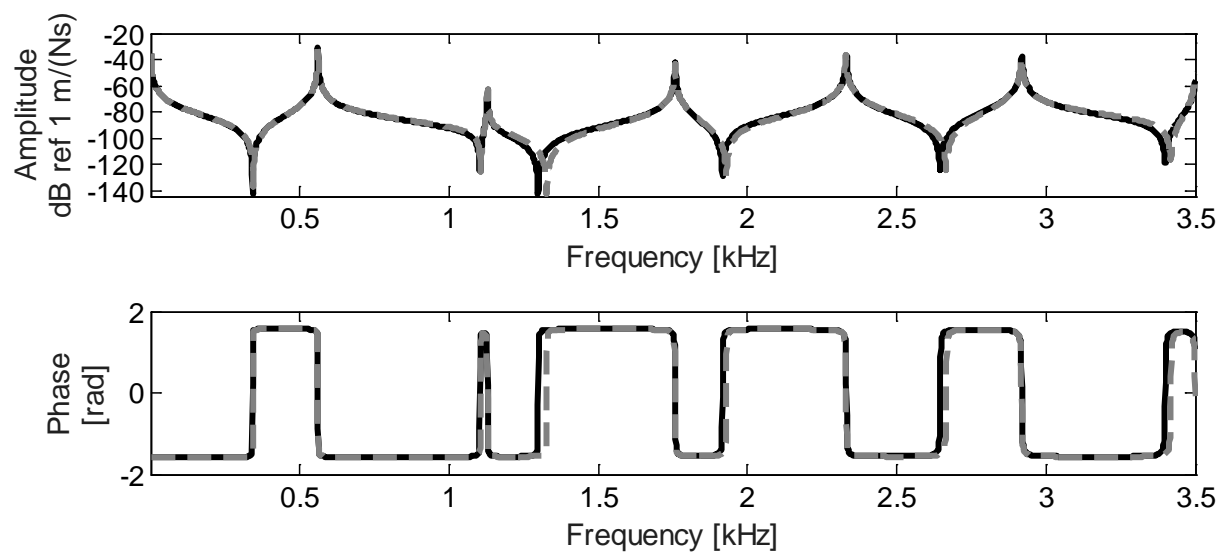

Figure 3. Amplitude and phase of the rod input mobility from FE (black solid line) and HFE (grey dashed line) for a single sample, using (a) $\delta_{E}=0.1, b=2 L$, (b) $\delta_{E}=0.1$, $b=0.8 \mathrm{~L}$ and (c) $\delta_{E}=0.15, b=0.2 \mathrm{~L}$. 
(a)

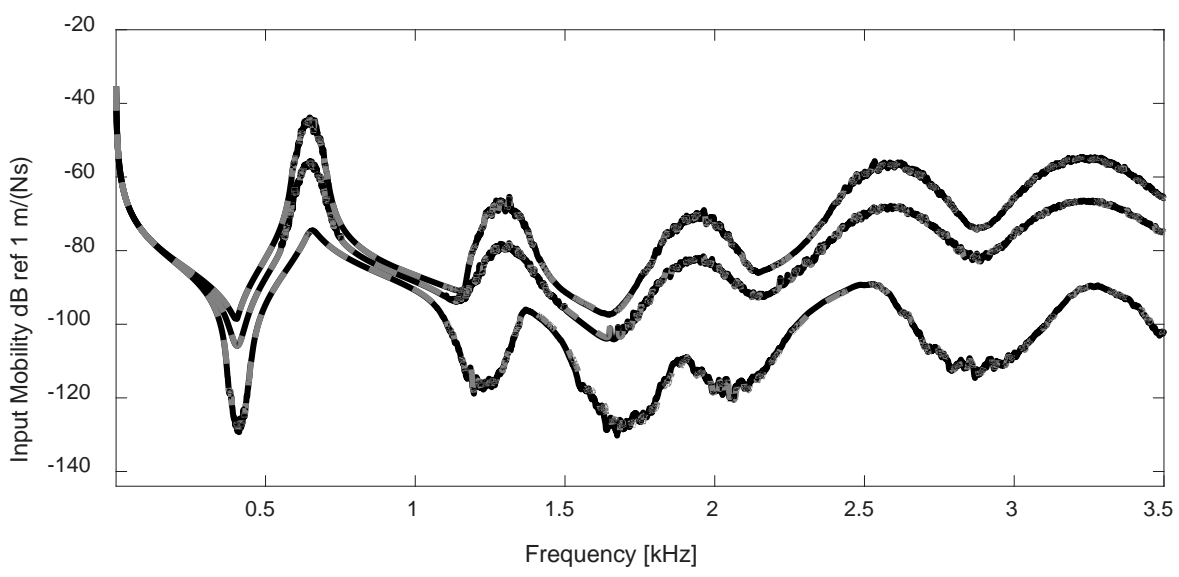

(b)

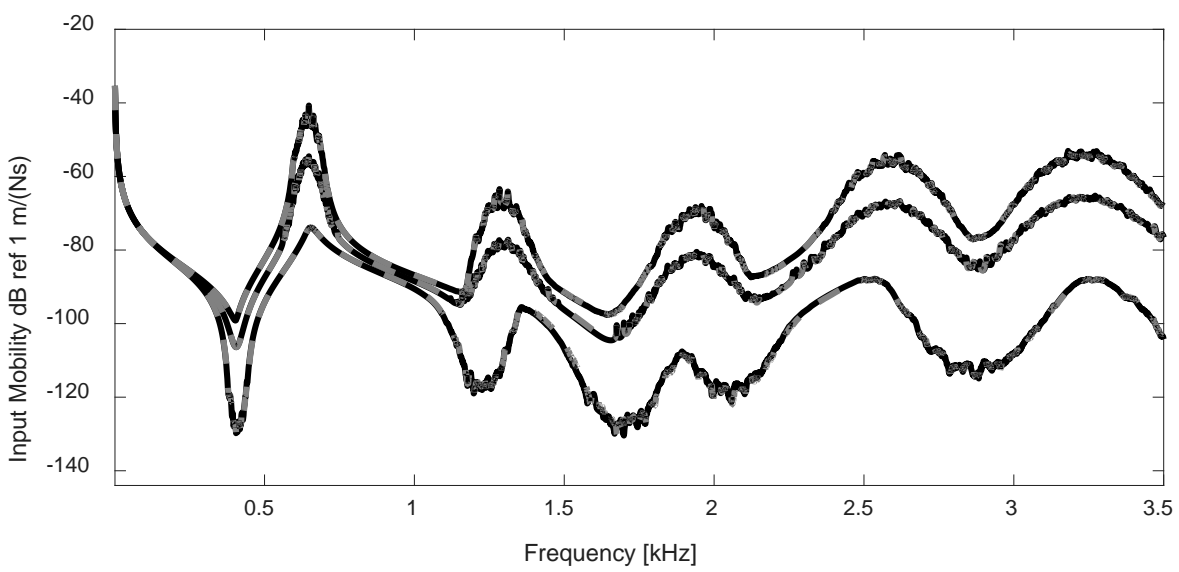

(c)

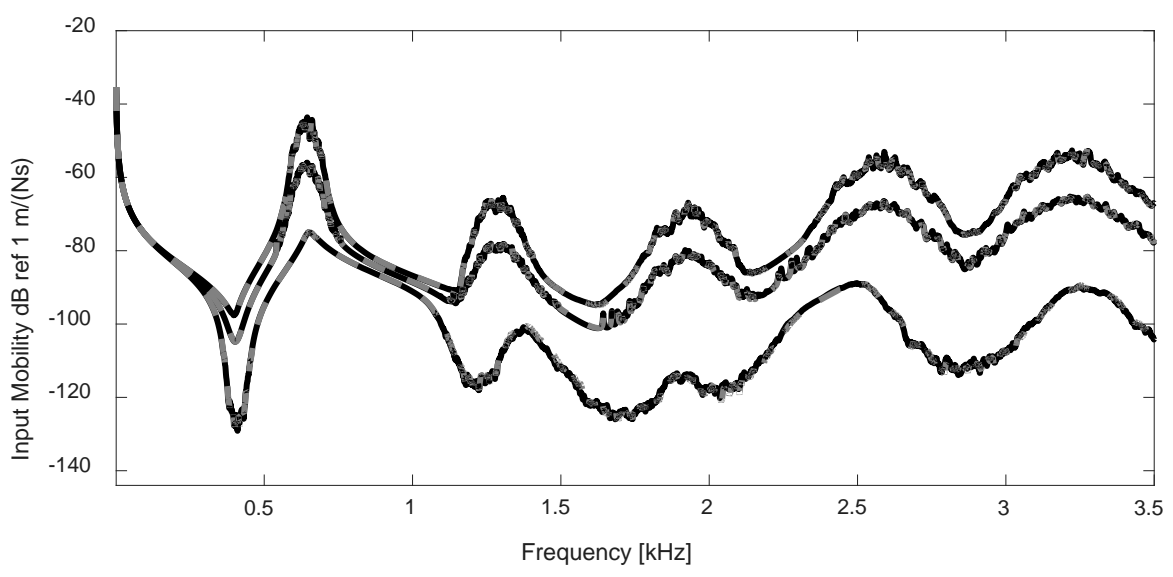

Figure 4. $5^{\text {th }}$ and $95^{\text {th }}$ percentile and mean value of the input mobility of the rod from FE (black solid) and HFE (grey dashed), using (a) $\delta_{E}=0.1, b=2 L$, (b) $\delta_{E}=0.1, b=$ $0.8 \mathrm{~L}$ and (c) $\delta_{E}=0.15, b=0.2 \mathrm{~L}$. 
(a)

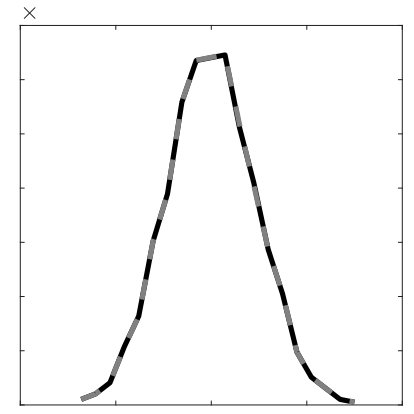

$\omega \omega$ (b)

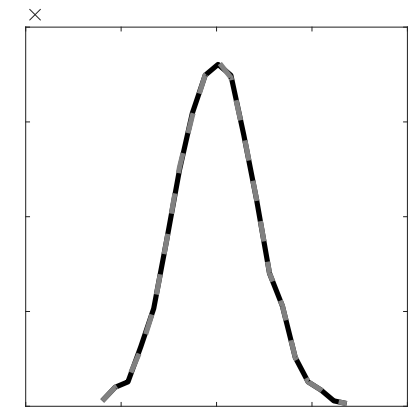

$\omega \omega$ (c)

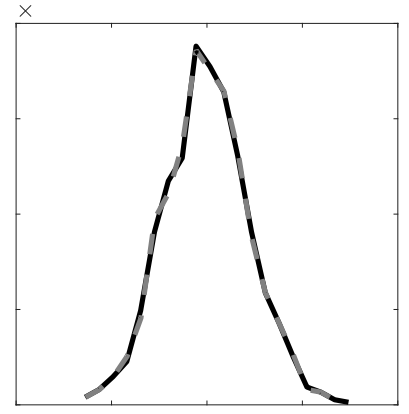

$\omega \omega$

Figure 5. PDF of the $4^{\text {th }}$ natural frequency $\omega_{4}$ of the rod from FE (black) and HFE (grey), using (a) $\delta_{E}=0.1, b=2 L$, (b) $\delta_{E}=0.1, b=0.8 L$ and (c) $\delta_{E}=0.15, b=$ $0.2 L$, where $\omega_{04}$ is the mean value of the natural frequency.

\subsection{Beam}

In this case, a beam with a rectangular cross-section of thickness $1 \mathrm{~mm}$ and width $30 \mathrm{~mm}$, i.e. $A=30 \mathrm{~mm}^{2}$ and total length $L=1 \mathrm{~m}$, with free-free boundary conditions is considered. The frequency band under analysis is up to $200 \mathrm{~Hz}$, discretised in $1 \mathrm{~Hz}$ steps, to include the first seven bending natural frequencies, and the excitation point is at $x=L_{1}=0.2 \mathrm{~L}$. Also, a standard FE approach with 100 elements is used for comparison. Additionally, the same random fields samples as in the rod case, shown in Figure 2, are used for calculating the input mobility, shown in Figure 6 for each considered $\delta_{E}$ and $b$ pair. The maximum order of the hierarchical functions was chosen to be $p=14$, using Eq. (8), similarly to the rod case, which leads to a total of 14 degrees of freedom while the FE model has 202 in total.

Figures 7 presents the mean value and $5^{\text {th }}$ and $95^{\text {th }}$ percentiles of the input mobility and Figure 8 shows the PDFs of the $4^{\text {th }}$ natural frequency, typical results, for each pair of $b$ and $\delta_{E}$. Response statistics of both HFE and FE approaches are calculated using the same random field samples. A very good agreement was observed in all of the cases, similar to the rod case. 
(a)
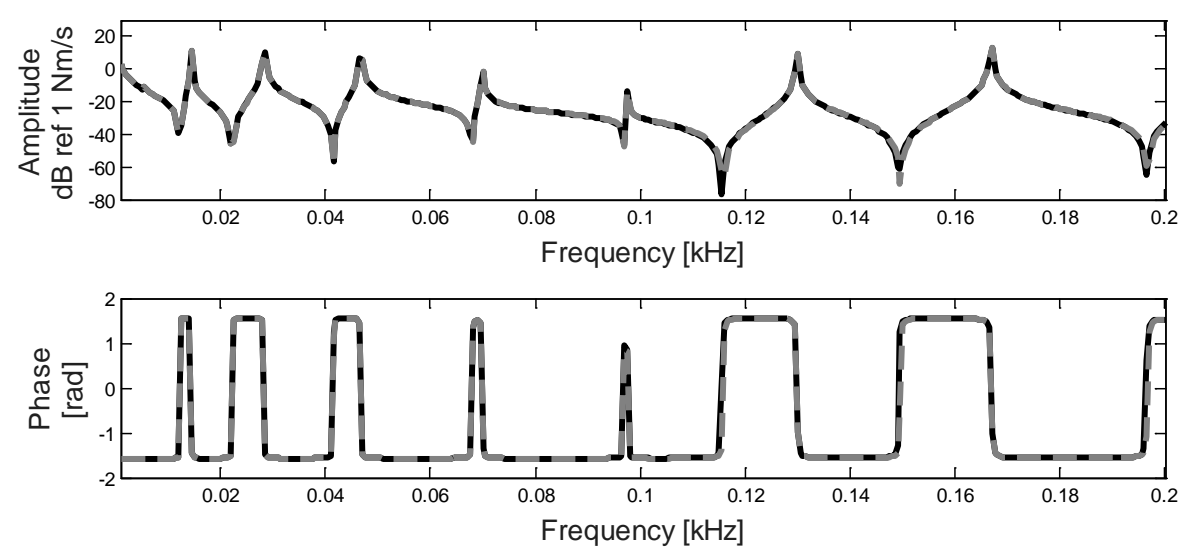

(b)
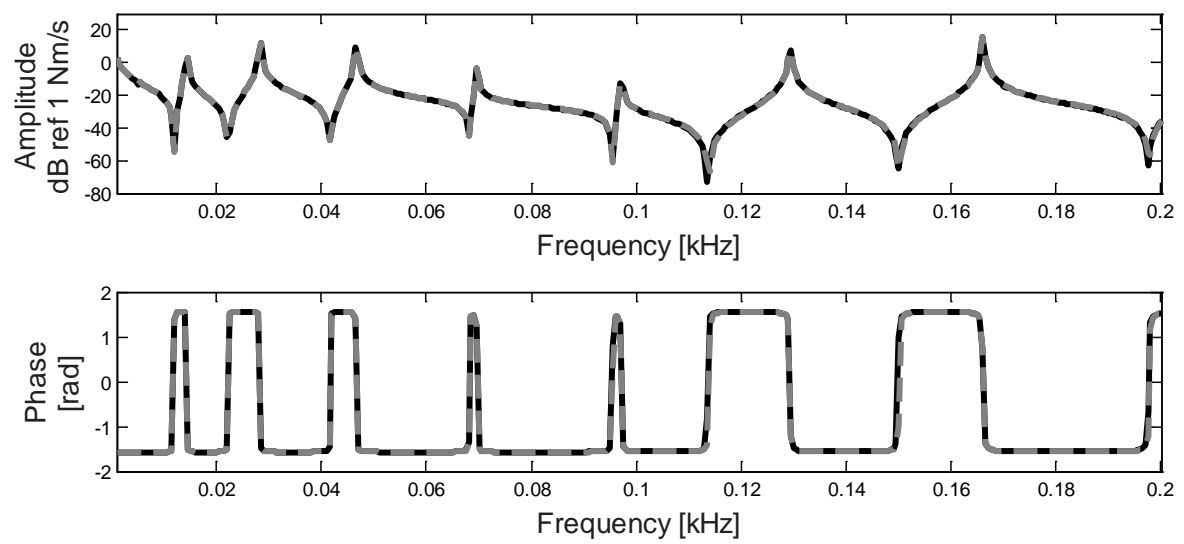

(c)
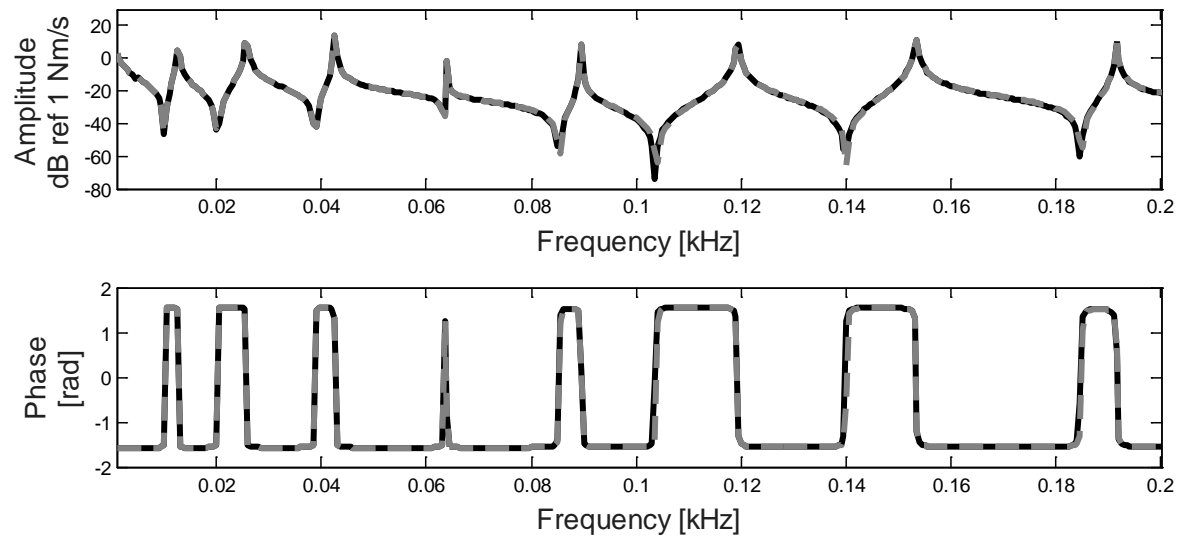

Figure 6. Amplitude and phase of the beam input mobility from FE (black solid line) and HFE (grey dashed line) for a single sample, using (a) $\delta_{E}=0.1, b=2 L$, (b) $\delta_{E}=$ $0.1, b=0.8 L$ and (c) $\delta_{E}=0.15, b=0.2 L$. 
(a)

(b)

(c)

Figure $7.5^{\text {th }}$ and $95^{\text {th }}$ percentile and mean value of the input mobility of the beam from FE (black solid) and HFE (grey dashed), using (a) $\delta_{E}=0.1, b=2 L$, (b) $\delta_{E}=0.1$, $b=0.8 \mathrm{~L}$ and (c) $\delta_{E}=0.15, b=0.2 \mathrm{~L}$. 
(a)

(b)

(c)

Figure 8. PDF of the $4^{\text {th }}$ natural frequency $\omega_{4}$ of the beam from FE (black) and HFE (grey), using (a) $\delta_{E}=0.1, b=2 L$, (b) $\delta_{E}=0.1, b=0.8 L$ and (c) $\delta_{E}=0.15, b=$ $0.2 L$, where $\omega_{04}$ is the mean value of the natural frequency.

\subsection{Plate}

This case concerns a rectangular plate with thickness $t=3 \mathrm{~mm}$, sides of length $L_{x}=1$ $\mathrm{m}$ and $L_{y}=0.75 \mathrm{~m}$, and simply supported at all of the edges, with the excitation point at $x=L_{1 x}=0.125 L_{x}$ and $y=L_{1 y}=1 / 3 L_{y}$. The frequency range up to $110 \mathrm{~Hz}$, discretised in $1 \mathrm{~Hz}$ steps, is considered and includes the first five natural frequencies. The natural frequencies of a plate with the mean homogeneous properties are given by $\omega_{11}$, $\omega_{21}, \omega_{12}, \omega_{31}$ and $\omega_{22}$, where the subscripts represent the number of half-waves in the $x$ and $y$ directions, respectively, of each mode. The plate FE model is meshed with 48 elements in the $x$ direction and 48 elements in $y$ direction, i.e., 2304 elements and 6823 degrees of freedom in total.

For the plate, the correlation lengths in the $x$ and $y$ direction are set to be equal, i.e. $b=$ $b_{x}=b_{y}$ and the same dispersion $\delta_{E}$ and correlation length $b$ as in the rod and beam cases. Samples of the normalized Young's modulus random field are shown in Figure 9 for each pair $\delta_{E}$ and $b$ considered.

Different to the previous cases, the input mobility is calculated by modal summation, such that the first 14 modes used were enough for adequate convergence, and is shown in Figure 10. For the plate element, using Eq. (9), a two-dimensional integral is required and the value $p_{r}=p_{s}=12$ was chosen, which is enough to achieve acceptable convergence of the natural frequencies in the frequency band under analysis. The simply supported boundary condition is applied by removing the corresponding hierarchical functions giving a total of 100 degrees of freedom.

Figures 11 presents the mean value and $5^{\text {th }}$ and $95^{\text {th }}$ percentiles of the input mobility and Figure 12 shows the PDFs of the natural frequency $\omega_{21}$, which is a typical result, for each pair $\delta_{E}$ and $b$ considered. Response statistics of both HFE and FE approaches are calculated using the same random field samples. A very good agreement was achieved in all of the cases, similar to the rod and beam cases.

In general, the statistics of the natural frequencies and forced response calculated using both approaches agree very well, although the HFE method requires only a fraction of the 
computational time of the FE approach. The most time-consuming step in the HFE formulation is the integration required to calculate the matrices $\Delta \mathbf{K}_{e}$ and $\Delta \mathbf{M}_{e}$. This, however, is done only once in the process, and the calculated matrices are the same for each MC sample. This drastically reduces the time spent for the multiple analysis required during statistical sampling. For plate-like structures or assemblies, the number of elements in a standard FE model can easily reach thousands and the computational cost can effectively make a stochastic analysis unfeasible or excessively expensive. Figure 13 shows the normalized computational cost of a typical simulation time as a function of the MC sample size for the analysis of the plate. The time is normalized by the total time taken for $1000 \mathrm{MC}$ samples to be calculated using the standard FE approach. Simulations were run on a Core i7 at $2.40 \mathrm{GHz}$ and 8GB RAM computer using a Matlab implementation. While the HFE method requires significant cost to set up the problem and compute $\Delta \mathbf{K}_{\mathbf{e}}$ and $\Delta \mathbf{M}_{\mathbf{e}}$, the solution time per subsequent analysis is, for this example, about $10^{3}$ times faster than the FE approach. Therefore, the curve for the HFE time in Figure 13 also includes the time component $t_{1}$ expended for the calculation of the $\Delta \mathbf{K}_{\mathbf{e}}$ and $\Delta \mathbf{M}_{\mathbf{e}}$ matrices, which is done only once during the sampling, and the time $t_{2}$ expended for sampling and solving for the forced response. It can be noticed that $t_{1}$ is smaller than the FE simulation for one single sample. Obviously, $t_{1}$ will increase for an increasing number of terms in the KL expansion, i.e. for shorter correlation lengths. Furthermore, this formulation could benefit from other methods for accelerating the convergence of the MC sampling, such as, for instance, line sampling [55]. The HFE approach itself is less time consuming for calculating the dynamic response of each sample when compared to the standard FE approach. 
(a)

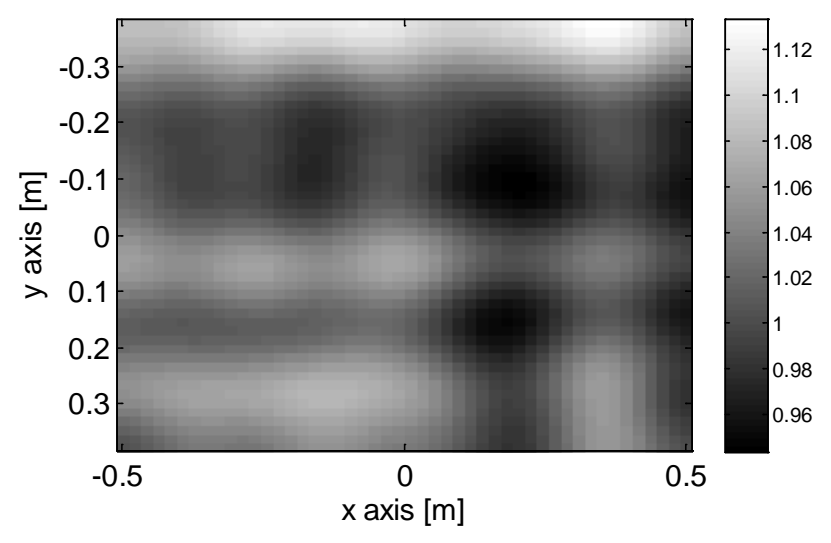

(b)

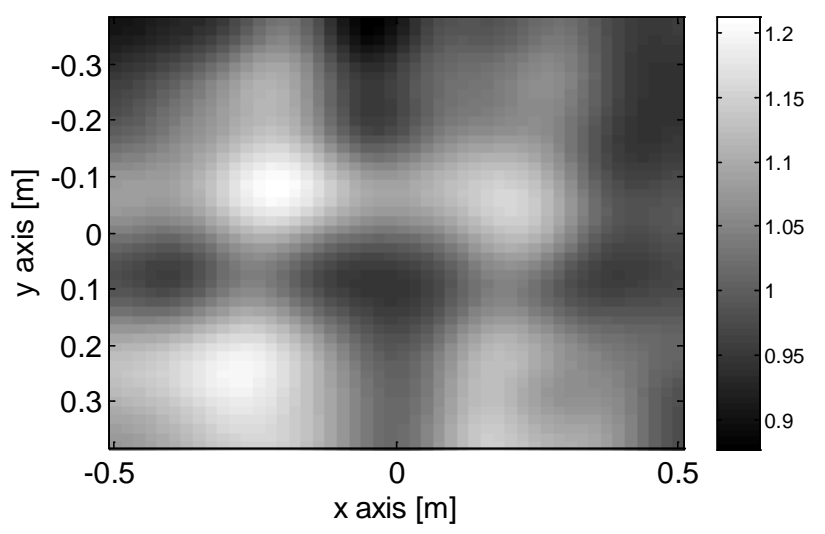

(c)

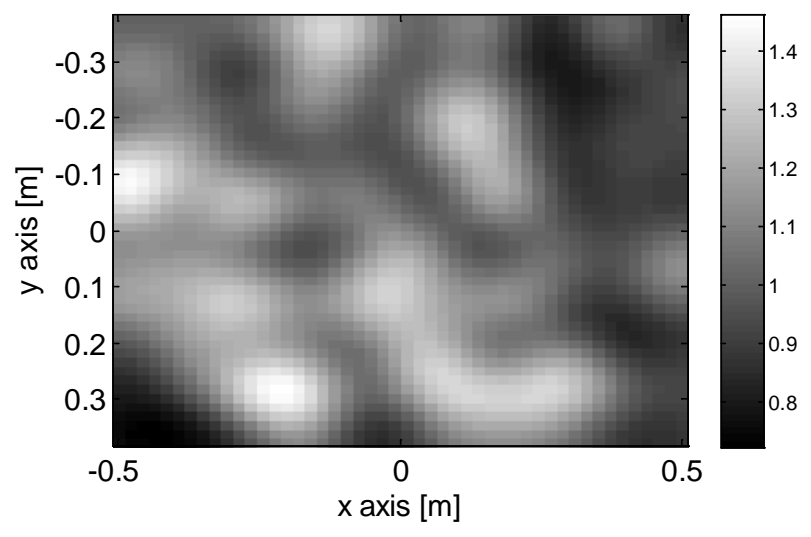

Figure 9. Samples of normalized Young's modulus $E(x, y) / E_{0}$ as a function of the position used for the plate for the cases of dispersion parameter $\delta_{E}$ and correlation length $b_{x}$ and $b_{y}$ (a) $\delta_{E}=0.1, b_{x}=b_{y}=2 L$, (b) $\delta_{E}=0.1, b_{x}=b_{y}=0.8 L$ and (c) $\delta_{E}=0.15, b_{x}=b_{y}=0.2 L$. 
(a)
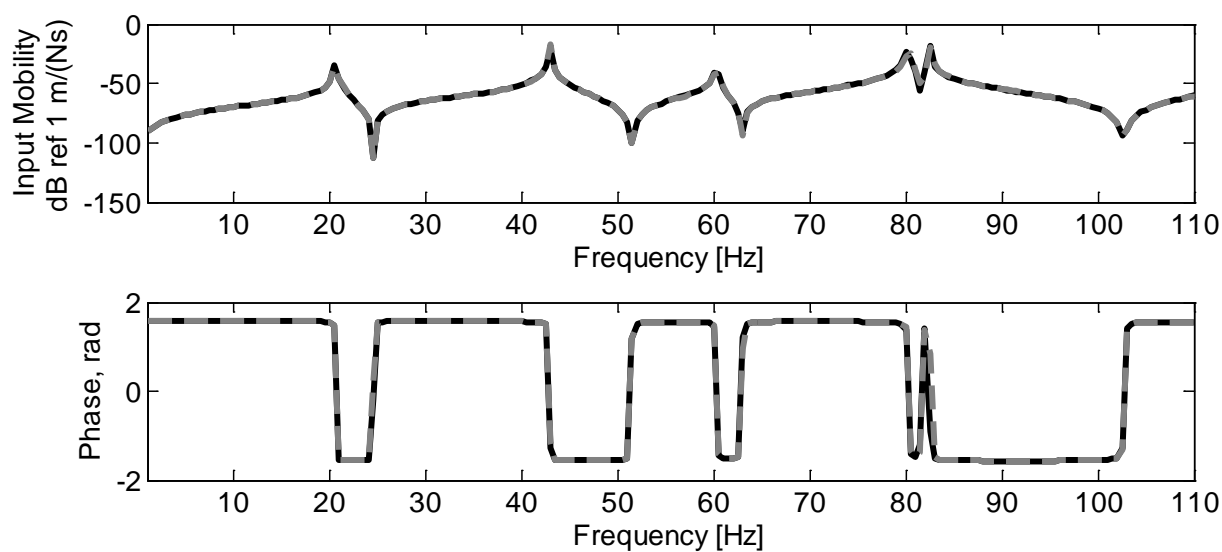

(b)
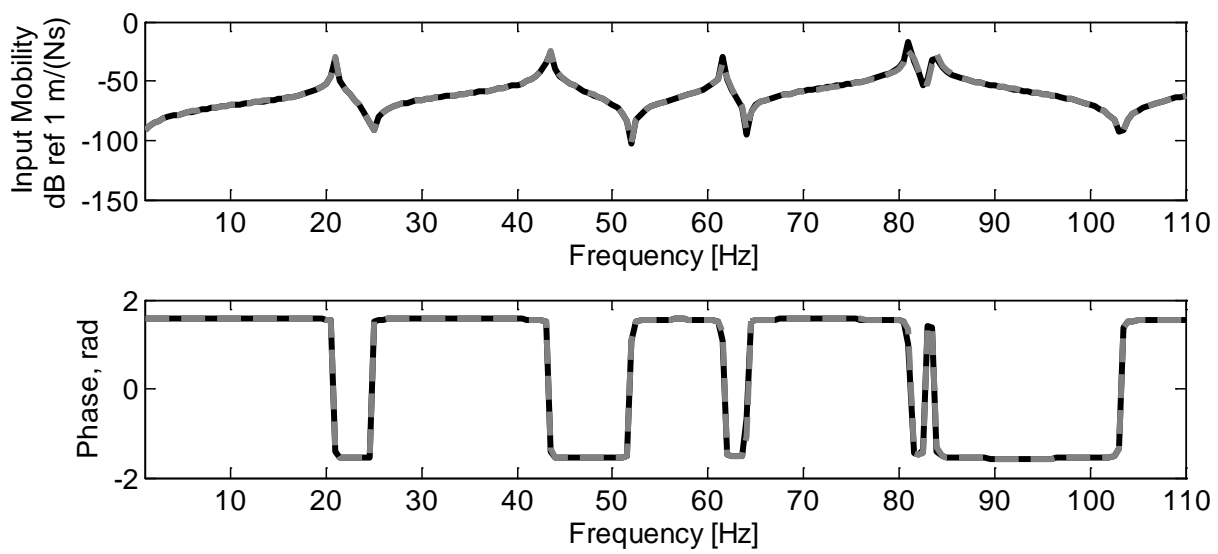

(c)
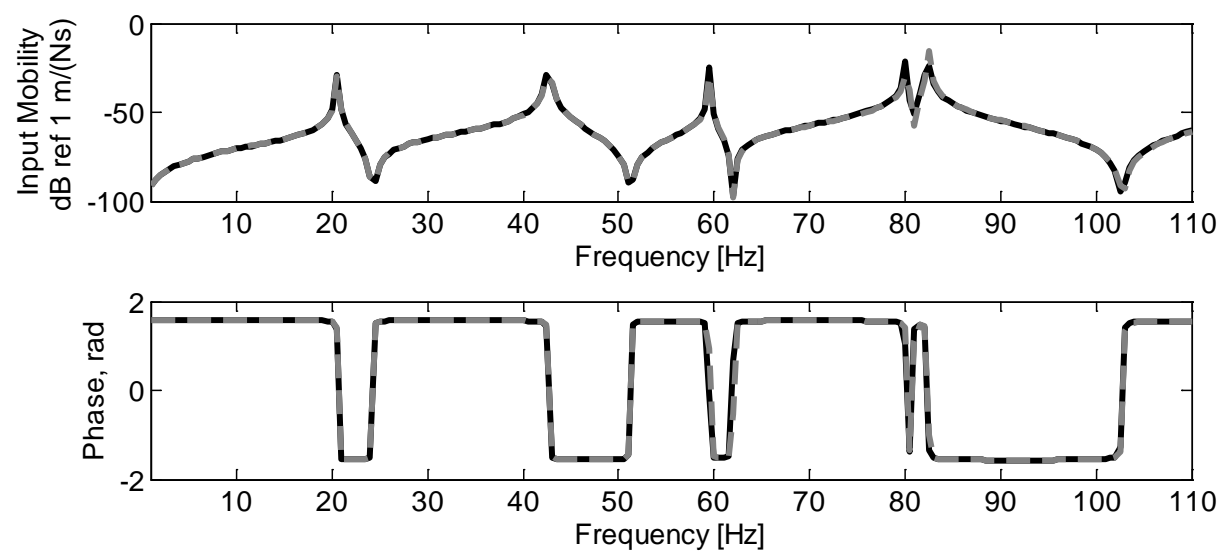

Figure 10. Amplitude and phase of the plate input mobility from FE (black solid line) and HFE (grey dashed line) for a single sample, using (a) $\delta_{E}=0.1, b_{x}=b_{y}=2 L$, (b) $\delta_{E}=0.1, b_{x}=b_{y}=0.8 \mathrm{~L}$ and (c) $\delta_{E}=0.15, b_{x}=b_{y}=0.2 \mathrm{~L}$. 
(a)

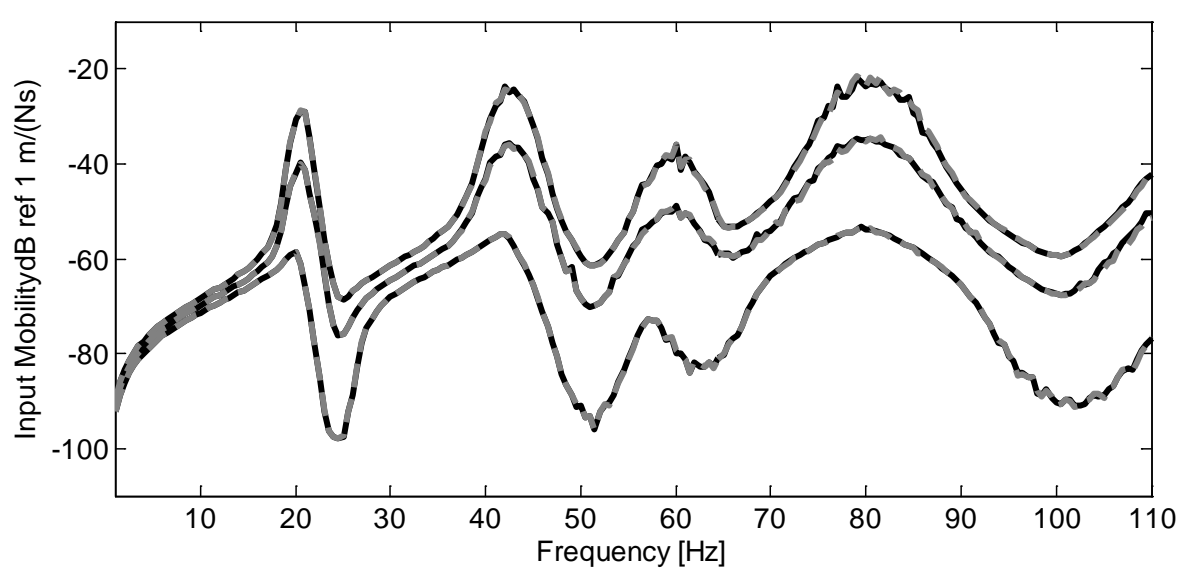

(b)

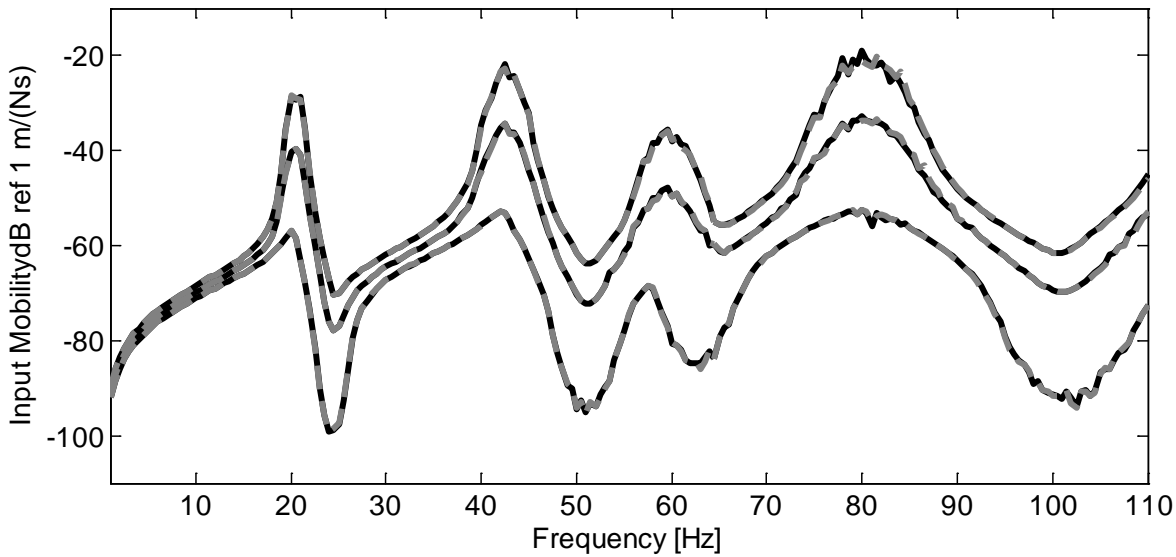

(c)

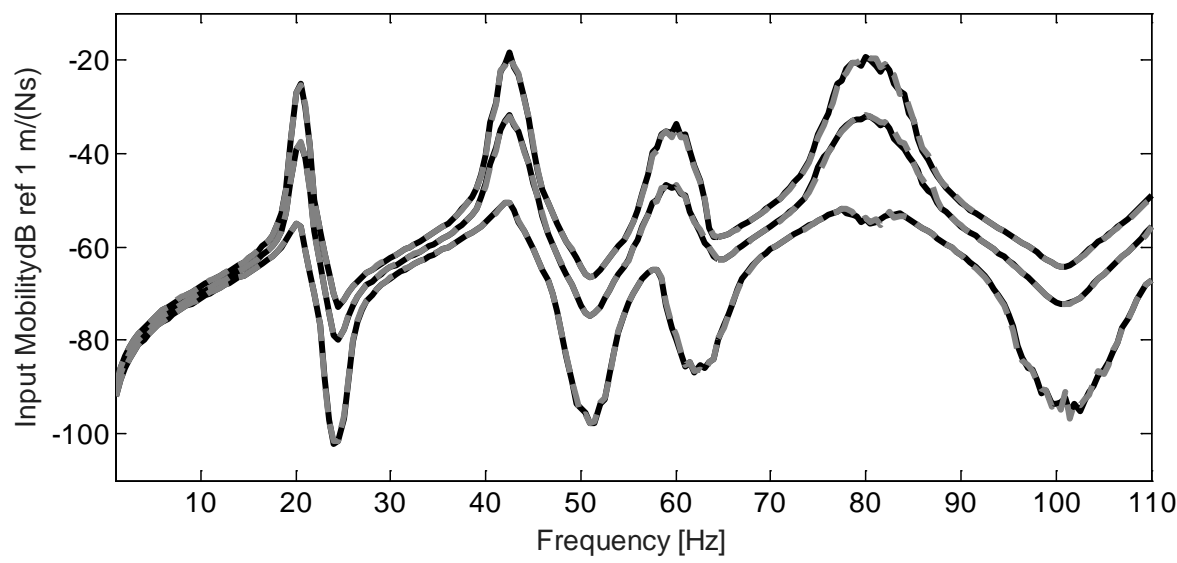

Figure $11.5^{\text {th }}$ and $95^{\text {th }}$ percentile (solid line) and mean value (dashed) of the input mobility of the plate from FE (black) and HFE (grey), using (a) $\delta_{E}=0.1, b=2 L$, (b) $\delta_{E}=0.1, b=0.8 L$ and (c) $\delta_{E}=0.15, b=0.2 L$. 
(a)

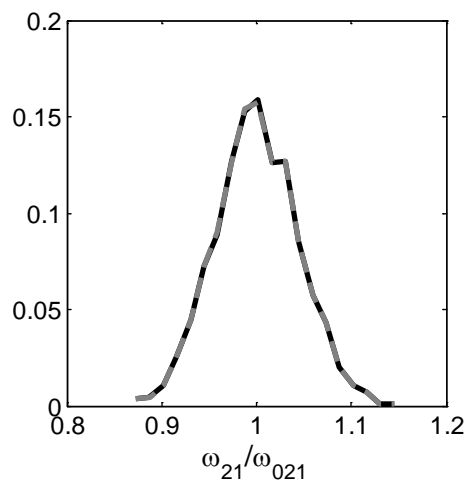

(b)

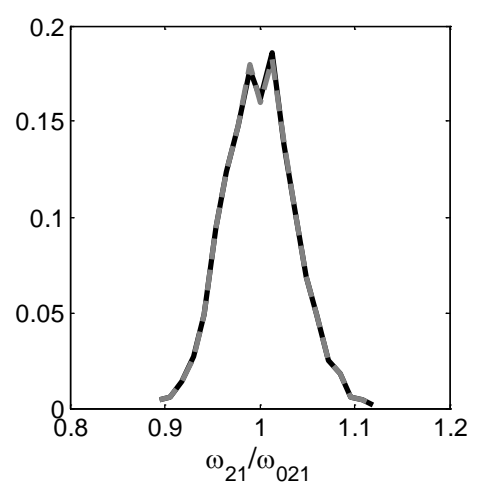

(c)

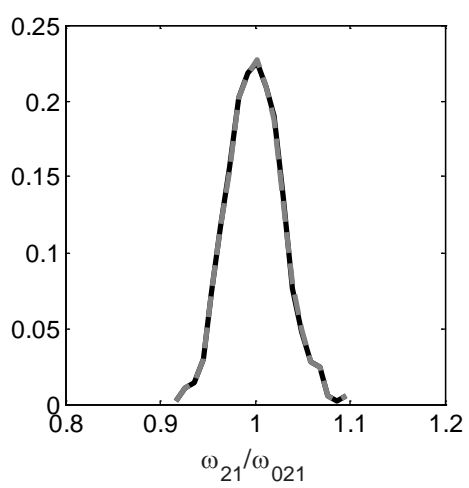

Figure 12. PDF of natural frequency $\omega_{21}$ of the plate from FE (black) and HFE (grey), using (a) $\delta_{E}=0.1, b=2 L$, (b) $\delta_{E}=0.1, b=0.8 L$ and (c) $\delta_{E}=0.15, b=0.2 L$, where $\omega_{021}$ is the mean value of the natural frequency.

Figure 13. Typical normalized simulation time in log scale for a plate as a function of the number of MC samples for the standard FE (black line) and HFE (dashed grey) approaches.

\section{Concluding remarks}

In this paper a numerical approach for the vibration analysis of structures with uncertain parameters using an enriched hierarchical basis, i.e. a Hierarchical Finite Element, method was presented. Spatial variability in the properties of the finite element was included in the element formulation. Throughout this paper, an analytical expression for a homogenous random field, with a specific correlation function, was used to model the random spatial variability. The formulation is not restricted to this description, however, as it allows for purely numerical solutions or analytical formulations of other correlation models to be incorporated.

The series representation of the random field was used in the HFE formulation to derive the stiffness and mass matrices for each term of the expansion. This procedure, although computationally expensive, has to be done only once, and then the random coefficients of the expansion can be sampled in a Monte Carlo framework to calculate the response 
statistics. Non-Gaussian distributions can be used by simply updating the random coefficients of the series, using an iterative scheme, maintaining the eigenfunctions of the Karhunen-Loève (KL) expansion. This avoids repeating the element integration procedure, which can be computationally costly and is particularly efficient for slowly correlated random fields, because fewer terms in the expansion are required.

The number of hierarchical functions used in the element integration has to take into account not only the usual frequency requirements, i.e. the number of half-waves in the highest order hierarchical function has to be greater than the number of half-waves in the structural modes, but also the random fluctuations of the spatially correlated properties. The maximum spatial frequency content of the random properties is thus used to determine this number, although further work is necessary to address the convergence of the method for other families of correlation functions and hierarchical functions for 1D and 2D structures.

A stochastic FE implementation, in which the KL series was discretized on the mesh using the mid-point method, was used for comparison of the numerical results. The $5^{\text {th }}$ and $95^{\text {th }}$ percentile envelopes of the input mobility show a very good agreement with full FE analysis as do estimates of the PDFs of the natural frequencies.

Results show that the method has potential application to the analysis of built-up structures with random, spatially correlated variability.

\section{Acknowledgements}

The authors gratefully acknowledge the financial support of the Brazilian National Council of Research (CNPq) Process number 445773/2014-6, the Federal District Research Foundation (FAPDF) Process number 0193001040/2015 and the Royal Society for the Newton International Exchanges Fund reference number IE140616.

\section{References}

[1] M. Petyt, Introduction to Finite Element vibration analysis, 2nd ed., Cambridge University Press, New York, USA, 2010.

[2] O.C. Zienkiewicz, K. Morgan, Finite Elements and Approximation, Dover Ed edition, Dover Publications, Mineola, N.Y., 2006.

[3] R. Ohayon, C. Soize, Structural acoustic and vibration: mechanical models, variational formulations and discretization, Academic Press, San Diego, CA, 1998.

[4] A. Deraemaeker, I. Babuška, P. Bouillard, Dispersion and pollution of the FEM solution for the Helmholtz equation in one, two and three dimensions, International Journal for Numerical Methods in Engineering. 46 (1999) 471-499. doi:10.1002/(sici)10970207(19991010)46:4<471::aid-nme684>3.0.co;2-6.

[5] L. Hinke, L. Pichler, H.J. Pradlwarter, B.R. Mace, T.P. Waters, Modelling of spatial variations in vibration analysis with application to an automotive windshield, Finite Elements in Analysis and Design. 47 (2011) 55-62. doi:10.1016/j.finel.2010.07.013.

[6] M.W. Zehn, A. Saitov, How can spatially distributed uncertainties be included in FEA and in parameter estimation for model updating?, Shock and Vibration. 10 (2003) 15-25.

[7] M. Gangadhar, M.W. Zehn, A methodology to model spatially distributed uncertainties in thin-walled structures, ZAMM - Journal of Applied Mathematics and Mechanics / Zeitschrift Für Angewandte Mathematik Und Mechanik. 87 (2007) 360-376. doi:10.1002/zamm.200610321. 
[8] J. Guilleminot, C. Soize, D. Kondo, C. Binetruy, Theoretical framework and experimental procedure for modelling mesoscopic volume fraction stochastic fluctuations in fiber reinforced composites, International Journal of Solids and Structures. 45 (2008) 5567-5583. doi:10.1016/j.ijsolstr.2008.06.002.

[9] A.T. Fabro, N.S. Ferguson, J.M. Gan, B.R. Mace, S. Bickerton, M. Battley, Estimation of random field material properties for chopped fibre composites and application to vibration modelling, Composite Structures. 125 (2015) 1-12. doi:10.1016/j.compstruct.2015.01.036.

[10] M.R. Machado, S. Adhikari, J.M.C. Dos Santos, J.R.F. Arruda, Estimation of beam material random field properties via sensitivity-based model updating using experimental frequency response functions, Mechanical Systems and Signal Processing. 102 (2018) 180-197. doi:10.1016/j.ymssp.2017.08.039.

[11] Z. Hu, S. Mahadevan, Uncertainty quantification and management in additive manufacturing: current status, needs, and opportunities, Int J Adv Manuf Technol. 93 (2017) 2855-2874. doi:10.1007/s00170-017-0703-5.

[12] E. Vanmarcke, Random Field: Analysis and Synthesis, 2nd Revised and Expanded, Word Scientific, Cambridge, MA, 2010.

[13] R. Ghanem, P.D. Spanos, Stochastic Finite Elements: A Spectral Approach, Revised edition, Dover Publications, Minneola, N.Y., 2012.

[14] M.M. Kaminski, Computational Mechanics of Composite Materials: Sensitivity, Randomness and Multiscale Behaviour, Springer, London, 2005.

[15] M. Ostoja-Starzewski, Material spatial randomness: From statistical to representative volume element, Probabilistic Engineering Mechanics. 21 (2006) 112-132. doi:10.1016/j.probengmech.2005.07.007.

[16] M. Ostoja-Starzewski, Stochastic finite elements: Where is the physics?, Theoretical and Applied Mechanics. 38 (2011) 379-396. doi:10.2298/TAM1104379O.

[17] D. Savvas, G. Stefanou, M. Papadrakakis, Determination of RVE size for random composites with local volume fraction variation, Computer Methods in Applied Mechanics and Engineering. 305 (2016) 340-358. doi:10.1016/j.cma.2016.03.002.

[18] A. Der Kiureghian, J.-B. Ke, The stochastic finite element method in structural reliability, Probabilist Engineering Mechanics. 3 (1988) 83-91.

[19] B. Sudret, A. Der Kiuereghian, Stochastic Finite Element methods and reliability: A stateof-Art report, University of California, Berkeley, 2000.

[20] A. Haldar, S. Mahadevan, Reliability Assessment Using Stochastic Finite Element Analysis, John Wiley \& Sons Inc., United States, 2000.

[21] G.I. Schuëller, H.J. Pradlwarter, Uncertain linear systems in dynamics: Retrospective and recent developments by stochastic approaches, Engineering Structures. 31 (2009) 25072517. doi:10.1016/j.engstruct.2009.07.005.

[22] G. Stefanou, The stochastic finite element method: Past, present and future, Computer Methods in Applied Mechanics and Engineering. 198 (2009) 1031-1051. doi:10.1016/j.cma.2008.11.007.

[23] J.D. Arregui-Mena, L. Margetts, P.M. Mummery, Practical Application of the Stochastic Finite Element Method, Archives of Computational Methods in Engineering. 23 (2016) 171-190. doi:10.1007/s11831-014-9139-3.

[24] M. Ostoja-Starzewski, A. Woods, Spectral finite elements for vibrating rods and beams with random field properties, Journal of Sound and Vibration. 268 (2003) 779-797. doi:10.1016/S0022-460X(03)00037-3.

[25] S. Adhikari, Doubly Spectral Stochastic Finite-Element Method for Linear Structural Dynamics, Journal of Aerospace Engineering. 24 (2011) 264-276. doi:10.1061/(ASCE)AS.1943-5525.0000070.

[26] L. Yang, Y. Zhou, J. Zhou, M. Wang, Hierarchical stochastic finite element method for structural analysis, Acta Mechanica Solida Sinica. 26 (2013) 189-196. doi:10.1016/S08949166(13)60018-X.

[27] N.S. Bardell, The free vibration of skew plates using the hierarchical finite element method, Computers \& Structures. 45 (1992) 841-874. doi:10.1016/0045-7949(92)90044-Z. 
[28] A. Houmat, An Alternative Hierarchical Finite Element Formulation Applied to Plate Vibrations, Journal of Sound and Vibration. 206 (1997) 201-215. doi:10.1006/jsvi.1997.1076.

[29] W. Han, M. Petyt, Linear vibration analysis of laminated rectangular plates using the hierarchical finite element method-I. Free vibration analysis, Computers \& Structures. 61 (1996) 705-712. doi:10.1016/0045-7949(95)00379-7.

[30] M Wachulec, P. H. Kirkegaard, Energy Flow in Plate Assembles by Hierarchical Version of Finite Element Method, Department of Civil Engineering, Aalborg University. (2001) 22.

[31] M Wachulec, Power Flow and Structure-Borne Noise in Medium Frequency Range, PhD Thesis, Department of Civil Engineering, Aalborg University, 2001.

[32] N.S. Bardell, G.J. Gange, An efficient static analysis of sandwich beams, Composite Structures. 29 (1994) 107-117. doi:10.1016/0263-8223(94)90040-X.

[33] Z. Yu, X. Guo, F. Chu, A multivariable hierarchical finite element for static and vibration analysis of beams, Finite Elements in Analysis and Design. 46 (2010) 625-631. doi:10.1016/j.finel.2010.03.002.

[34] G. Giunta, S. Belouettar, H. Nasser, E.H. Kiefer-Kamal, T. Thielen, Hierarchical models for the static analysis of three-dimensional sandwich beam structures, Composite Structures. 133 (2015) 1284-1301. doi:10.1016/j.compstruct.2015.08.049.

[35] A. Boukhalfa, A. Hadjoui, Free vibration analysis of an embarked rotating composite shaft using the hp-version of the FEM, Latin American Journal of Solids and Structures. 7 (2010) 105-141. doi:10.1590/S1679-78252010000200002.

[36] W. Han, M. Petyt, Linear vibration analysis of laminated rectangular plates using the hierarchical finite element method-II. Forced vibration analysis, Computers \& Structures. 61 (1996) 713-724. doi:10.1016/0045-7949(96)00213-1.

[37] G. Giunta, D. Crisafulli, S. Belouettar, E. Carrera, Hierarchical theories for the free vibration analysis of functionally graded beams, Composite Structures. 94 (2011) 68-74. doi:10.1016/j.compstruct.2011.07.016.

[38] Y. Hui, G. Giunta, S. Belouettar, Q. Huang, H. Hu, E. Carrera, A free vibration analysis of three-dimensional sandwich beams using hierarchical one-dimensional finite elements, Composites Part B: Engineering. 110 (2017) 7-19. doi:10.1016/j.compositesb.2016.10.065.

[39] R.Y. Rubinstein, D.P. Kroese, Simulation and the Monte Carlo method, Second Edition, John Wiley \& Sons, Inc., Hoboken, NJ, USA, 2007.

[40] W. Betz, I. Papaioannou, D. Straub, Numerical methods for the discretization of random fields by means of the Karhunen-Loève expansion, Computer Methods in Applied Mechanics and Engineering. 271 (2014) 109-129. doi:10.1016/j.cma.2013.12.010.

[41] S.P. Huang, S.T. Quek, K.K. Phoon, Convergence study of the truncated Karhunen-Loeve expansion for simulation of stochastic processes, International Journal for Numerical Methods in Engineering. 52 (2001) 1029-1043. doi:10.1002/nme.255.

[42] K.K. Phoon, S.P. Huang, S.T. Quek, Simulation of second-order processes using KarhunenLoeve expansion, Computers \& Structures. 80 (2002) 1049-1060. doi:10.1016/S00457949(02)00064-0.

[43] K.K. Phoon, H.W. Huang, S.T. Quek, Simulation of strongly non-Gaussian processes using Karhunen-Loeve expansion, Probabilistic Engineering Mechanics. 20 (2005) 188-198. doi:10.1016/j.probengmech.2005.05.007.

[44] L.B. Li, K.K. Phoon, S.T. Quek, Comparison between Karhunen-Loève expansion and translation-based simulation of non-Gaussian processes, Computers \& Structures. 85 (2007) 264-276. doi:10.1016/j.compstruc.2006.10.010.

[45] D.C. Charmpis, G.I. Schuëller, M.F. Pellissetti, The need for linking micromechanics of materials with stochastic finite elements: A challenge for materials science, Computational Materials Science. 41 (2007) 27-37. doi:10.1016/j.commatsci.2007.02.014.

[46] N.S. Bardell, Free vibration analysis of a flat plate using the hierarchical finite element method, Journal of Sound and Vibration. 151 (1991) 263-289. doi:10.1016/0022460X(91)90855-E. 
[47] D.L. Allaix, V.I. Carbone, Discretization of 2D random fields: A genetic algorithm approach, Engineering $\quad$ Structures. 31 (2009) 1111-1119. doi:10.1016/j.engstruct.2009.01.008.

[48] S. Shang, G.J. Yun, Stochastic finite element with material uncertainties: Implementation in a general purpose simulation program, Finite Elements in Analysis and Design. 64 (2013) 65-78. doi:10.1016/j.finel.2012.10.001.

[49] Y. Ma, Y. Zhang, D. Kennedy, Energy flow analysis of mid-frequency vibration of coupled plate structures with a hybrid analytical wave and finite element model, Computers \& Structures. 175 (2016) 1-14. doi:10.1016/j.compstruc.2016.06.007.

[50] E.C.N. Wester, B.R. Mace, Wave component analysis of energy flow in complex structures - Part I: a deterministic model, Journal of Sound and Vibration. 285 (2005) 209-227. doi:10.1016/j.jsv.2004.08.025.

[51] E.C.N. Wester, B.R. Mace, Wave component analysis of energy flow in complex structures-Part II: ensemble statistics, Journal of Sound and Vibration. 285 (2005) 229-250. doi:10.1016/j.jsv.2004.08.026.

[52] J. Legault, J. Woodhouse, R.S. Langley, Statistical energy analysis of inhomogeneous systems with slowly varying properties, Journal of Sound and Vibration. 333 (2014) 72167232. doi:10.1016/j.jsv.2014.08.026.

[53] A.T. Fabro, N.S. Ferguson, T. Jain, R. Halkyard, B.R. Mace, Wave propagation in onedimensional waveguides with slowly varying random spatially correlated variability, Journal of Sound and Vibration. 343 (2015) 20-48. doi:10.1016/j.jsv.2015.01.013.

[54] C. Li, A. Der Kiureghian, Optimal Discretization of Random Fields, Journal of Engineering Mechanics. 119 (1993) 1136-1154. doi:10.1061/(ASCE)0733-9399(1993)119:6(1136).

[55] P.S. Koutsourelakis, H.J. Pradlwarter, G.I. Schuëller, Reliability of structures in high dimensions, part I: algorithms and applications, Probabilistic Engineering Mechanics. 19 (2004) 409-417. doi:10.1016/j.probengmech.2004.05.001.

[56] W. Han, M. Petyt, Geometrically nonlinear vibration analysis of thin, rectangular plates using the hierarchical finite element method-I: The fundamental mode of isotropic plates, Computers \& Structures. 63 (1997) 295-308. doi:10.1016/S0045-7949(96)00345-8.

[57] A. Houmat, Nonlinear free vibration of a composite rectangular specially-orthotropic plate with variable fiber spacing, Composite Structures. 94 (2012) 3029-3036. doi:10.1016/j.compstruct.2012.05.006.

[58] C. Soize, Uncertainty Quantification - An Accelerated Course with Advanced Applications in Computational Engineering, 1st ed., Elsevier, 2017.

[59] M. Schevenels, G. Lombaert, G. Degrande, Application of the stochastic finite element method for Gaussian and non-Gaussian systems, in: Proceedings of ISMA2004, Leuven, Belgium, 2004. http://bwk.kuleuven.be/apps/bwm/papers/scheip04a.pdf.

[60] A. M. J. Olsson, G. E. Sandberg, Latin Hypercube Sampling for Stochastic Finite Element Analysis, Journal of Engineering Mechanics. 128 (2002). doi:10.1061/(ASCE)07339399(2002)128:1(121).

[61] B.A. Zeldin, P.D. Spanos, On random field discretization in Stochastic Finite Elements, Journal of Applied Mechanics. 65 (1998) 320-327. doi:10.1115/1.2789057. 